\title{
Age of Information Minimization of Multichannel Allocation Mechanism for Hybrid Cognitive Radio Networks with Energy Harvesting under Collusion Constraint
}

HAKAN MURAT KARACA ( $\nabla$ hakan.karaca@cbu.edu.tr )

Celal Bayar University

\section{Research}

Keywords: Cognitive radio networks, Energy harvesting, Age of information, Information freshness, Hybrid underlay/overlay scheme, Interference constraint

Posted Date: February 23rd, 2021

DOI: https://doi.org/10.21203/rs.3.rs-220122/v1

License: (c) (i) This work is licensed under a Creative Commons Attribution 4.0 International License. Read Full License 


\section{RESEARCH}

\section{Age of Information Minimization of Multichannel Allocation Mechanism for Hybrid Cognitive Radio Networks with Energy Harvesting under Collusion Constraint}

\author{
Hakan M Karaca*
}

\section{Correspondence:}

hakan.karaca@cbu.edu.tr

Department of Computer

Engineering, Celal Bayar

University, Muradiye, Manisa,

Turkey

Full list of author information is

available at the end of the article

${ }^{*}$ Equal contributor

\begin{abstract}
Cognitive radio networks (CRNs) with energy harvesting has been a promising solution for wireless industrial networks and seems a promising solution for the spectrum scarcity problem. However, it is critical the wireless spectrum should carefully be managed in order to fullfill the tight requirements on the trustworthy and minimum delayed delivery of information. Motivated by this, in order to represent the freshness of the information, this paper takes into account the metric of age of information (Aol) and multi-channel allocation problem in hybrid cognitive radio networks where secondary transmitters (STs) are able to harvest energy is investigated in terms of both maximization of allocation performance while minimizing average $\mathrm{AOI}$ values. A novel mechanism has been proposed to restrict average Aol values while distributing channels to STs by handling both collusion and energy constraints. STs are prioritized based on a proposed metric and they are able to obtain channels only if the resulting Aol value satisfy proposed conditions. Aol values are computed based on co-channel interference between STs and minimized by decreasing interference levels. For comparison, two algorithms were considered: a greedy mechanism for m-channel allocation of hybrid CRNs with harvesting, but without AOI control and the proposed $\mathrm{m}$-channel allocation schemes based on sorting STs according to the proposed metric and controlling AOI levels based on co-channel interference. The simulations depict that performance of the proposed m-channel allocation method outweighs the greedy algorithm in terms of both Aol minimization and maximization of channel allocation performance, proving the superiority of the proposed algorithm.
\end{abstract}

Keywords: Cognitive radio networks; Energy harvesting; Age of information; Information freshness; Hybrid underlay/overlay scheme; Interference constraint

\section{Introduction}

With the widespread use of wireless nodes, delivery of data on time has become crucial for various applications, like vehicle tracking, vehicle-to-vehicle networking, and natural disaster monitoring where status information should be periodically refreshed. Freshness of information in such situations necessitates to design a new network metric.

The concept of Age of Information (AoI) was first presented in 2011 in [1] to quantify the freshness of the information we have about the status of a remote system. More specifically, the age captures the time elapsed since the last received 
message containing update information. The AoI captures both the network delay and the generation time for each status update. If the most recently received status update carries the information sampled at time $\mathrm{r}(\mathrm{t})$, the status update age at time $\mathrm{t}$ can be defined as $\mathrm{t}-\mathrm{r}(\mathrm{t})$. This metric has attracted a brilliant interest over the last years, due to its originality to characterize the freshness of information in a communication system, that differs it from other common metrics such as delay. The first group of characterizations of the AoI metric had appeared by 2012 in some papers ([2], [3] and [4]) by deploying it in a simple communication system model. After that, AoI has drawn a growing interest, with over 50 publications.

Time-sensitive information in a monitoring system can be described by means of real-time status updates in the form of packets. Such packets should include (i) the status update information and (ii) information about when they are generated. Status updates may include sensor monitoring data, stock market data, and follow time-varying ingredients which should be carried from the location where created to a distant point within a network. The freshness of status update data was first studied in the context of vehicular networks $[2,3,4]$, where the authors worked on the broadcast problem. Specifically, sensor nodes catching speed, acceleration, parking radars measurements etc., distribute this time crucial information to interlinked transportations across the network to increase road safety and transportation efficiency [5]. Timeliness of data is a requirement in various other applications, for instance, wireless smart camera networks $[6,7]$, broadcast wireless networks [8], wireless sensor networks (WSN) for environmental screening and healthcare data $[9,10]$, active data repository [11], content caching $[12,13,14,15]$, real time databases [16], ad hoc networks $[17,18]$ and unmanned aerial vehicle (UAV)-assisted IoT networks [19]. In addition, in the field of adaptive transmission, important efficiency profit can be obtained by means of adaptive signalling techniques (e.g. modulation and coding) [20, 21]. On the other hand, this feedback mechanism is restricted by the acquisition of channel state information (CSI which causes much communication overhead, and also delayed CSI could decrease the performance of algorithms especially while channels quickly change in a dynamic environment. The term of timeliness as indicated by age can be investigated in various systems in the form of either a concept, a performance metric, or a tool. AoI can be modelled and used as a performance metric together with numerous relevant metrics derived in relation to it, for instance the peak age of information (PAoI) [22, 23], the cost of update delay (CoUD) [24, 25], also called as non-linear age penalty function [27], the value of information of update (VoIU) [24, 25, 28], the age of synchronization (AoS) [29], and the age of incorrect information (AoI) [30]. In older works on AoI, the investigation and definition of age has been possible by means of a queueing theoretic perspective [31, 32]. [31] studies $\mathrm{M} / \mathrm{M} / 1, \mathrm{M} / \mathrm{D} / 1, \mathrm{D} / \mathrm{M} / 1$ models and first-come-first-served (FCFS) queues. Recently, AoI has been introduced for energy harvesting scenarios, where harvested energy is used in each update [33, 34, 35, 36]. If updates are prevented as a result of lack of energy, information could stay outof-date. Therefore, in order to maintain update of information as fresh as possible, it is necessary to optimally handle energy. AoI has been first studied in [33] where an energy harvesting source is taken into account which carries out status updates using the harvested energy. In [33] they showed that based on the energy causality necessity AoI is improved by waiting between updates instead of updating as 
quickly as possible. Recent works employ the concept of AoI in energy harvesting communication systems (from natural renewable sources), and study transmission policies that minimize AoI-based performance metrics [37, 38, 39, 40].

In the cognitive radio $(\mathrm{CR})$ technology, a secondary system may access the spectrum formally allocated to a primary system, subject to little degradation introduced to the primary service. There are two main access strategies for the secondary system which are overlay and underlay schemes [41, 42, 43, 44]. In the overlay scheme, the secondary system senses spectrum and starts its transmission when the primary system is idle. In the underlay scheme, the secondary system can always access the spectrum subject to an interference constraint. However, it must always satisfy the interference constraint even if the primary system is idle [45].

The freshness of the status updates is important for both primary and secondary systems in a CR. For example, in smart parking applications, sensors check whether a parking space is occupied and report the results to the users. The timeliness of the updates from the sensor is important since the users are involved in the latest information about whether parking space is free or busy. In fact, the standard metric delay cannot specify the timeliness enough. Cognitive radio networks (CRN) with energy harvesting capability have been investigated with the throughput as the main metric [46, 47, 48]. In [46] and [47], the spectrum sensing and/or accessing choices are obtained by resolving partially observable Markov decision processes (POMDPs).

AoI minimization for a cognitive radio network (CRN) is handled in [49]. The AoI of the primary user is modelled and reduced by using a queuing theoretic perspective.

In another work [50], a finite-horizon sequential decision-making problem related to the energy constraint is solved in order to minimize the AoI of an energy harvesting secondary user in a CRN. Assuming perfect spectrum sensing at the secondary user, they derived an optimal sensing and status update mechanism. They examine optimal sensing and update decisions that reduce the average AoI over a finite horizon for a energy harvesting $\mathrm{CR}$ which requires to keep the information at its destination as up-to-date as possible.

AoI minimization for point-to-point communication with energy harvesting restrictions has been investigated in [33]. It is shown that AoI is decreased by waiting before update by optimizing of the inter-update time.

In [51], an energy harvesting cognitive secondary user with the aim of AoI minimization is proposed. To examine the impact of imperfect spectrum sensing on AoI minimization in the long period, an infinite horizon POMDP is developed.

An AoI aware channel allocation problem in a software defined network based wireless industrial networks is considered in [52]. The freshness of information delivered in the network is modelled using AoI and they formulated the AoI aware channel allocation problem as a non-linear integer programming problem. Two efficient heuristic algorithms are proposed as a solution which exploits the special structure of the problem.

In this journal, an ST can use two transmission modes: overlay and underlay. Its transmission mode depends on the distance to the primary transmitter, consequence of spectrum sensing activity and current energy level in the battery [53]. The PT is 
assumed to have a guard zone where an ST cannot make any transmission. Based on this, as defined in [54], the area of overlay mode begins where the coverage area of PT finishes, the underlay area is the area between the end of guard zone and end of the coverage area. None of STs can make transmission in the guard zone to protect its intended PT from ST interference. The harvesting zone (HZ) is the area where an ST can do harvesting from radio signals of an active PT. Harvesting is possible till a certain distance only because of attenuation.

Different from previous works, this paper studies both throughput maximization and AoI minimization at the same time for optimal m-channel allocation using the hybrid overlay/underlay mechanism in CRNs with energy harvesting capability under both the energy and collision constraints. It not only tries to reduce AoI, but also maximize channel allocation performance without disturbing primary user communication. For this purpose, a new algorithm is proposed where spectrum reuse was increased by enabling energy harvesting without disturbing the primary transmission when AoI is minimized. The energy limitation satisfies the harvested energy should be higher than the consumed energy, and ST should stop spectrum usage trials in cases where there is short of energy. For the collision constraint to be met, the interference caused by STs on primary transmitters (PTs) should be lower than a threshold for a certain signal to interference plus noise ratio. ST harvests energy from existing primary users' signals, like energy-harvesting CRNs in [55].

To the best of author' s knowledge, this is the first attempt to minimize AoI while maximizing $\mathrm{m}$-channel allocation performance in a hybrid CRN with energy harvesting capability. The main contributions of this article can be summarized as follows:

(1) For a CRN with mobile energy harvesting ST, this paper is the first attempt which tries both to minimize AoI and maximize m-channel allocation performance of the hybrid CRN under both collusion and energy constraints. So, allocation is done such that primary users are protected from ST interference and AoI is reduced.

(2) For allocation of channels, STs are prioritized based on their channel transmission and harvesting capabilities such that STs having higher transmission rate and harvesting capability will have more priority to have channel. For this purpose a metric is proposed and calculated at the beginning of each time interval. But most important contribution is, AoI value for each ST is calculated before allocating channel and decision of allocation is given checking proposed criterion. A few criteria are proposed, for example, when allocating a channel to an ST, if increase of AoI value will be more than $50 \%$ of previous value of AoI, the ST will not be able to obtain the channel. Another proposed criterion is at each internal, average AoI of all STs is calculated and the ST will be able to get the channel only if its new AoI value will be less than the calculated average one. For all those criterion, simulations are run and both allocation performance and AoI change versus time are compared.

(3) Simulations show that AoI values are markedly reduced when several proposed criterion are applied and compared. However, because of AoI constraint, channel distribution performance is also limited which means there is a trade off between them. Therefore, an optimum solution for both AoI and channel distribution performance can be found. Besides this, by harvesting energy from 
ambient RF signals when assigning channels, again channel distribution performance is improved, as proposed in [53]. As more number of users located in the defined HZ (density of SUs), the more users located in the HZ, which means more energy can be harvested, thereby increasing channel distribution performance. On the other hand, the performance considerably depends on the Poisson distribution density of SUs. The robustness against signal to interference and noise ratio (SINR) thresholds is also investigated and found that it AoI decreases with increasing SINR (outage) threshold as expected, since increasing SINR threshold creates more constraint on allocation. Moreover, detailed complexity analysis of all algorithms are provided and compared.

The rest of this paper is organized as follows. Section ?? describes the system model and notations which consists of 3 subsections. Section ?? introduces the greedy mechanism and proposed schemes. Simulation results and their comments are explained in Section ??. Section ?? gives the concluding remarks of this paper.

\section{Methods/Experimental}

0.1 Network modelling

A cognitive communications system is considered where energy harvesting SUs try to utilize spectrum without disturbing primary user communication. Also, AoI is tried to be minimized by checking corresponding AoI level before allocation of each channel. Spectrum sharing based on access technology can be implemented by either overlay or underlay access technique. In overlay access technique, SU should find a spectrum hole and allowed to use it without restrictions. On the other hand, if PU returns to the spectrum, SU has to leave the spectrum or change to another free channel. As a result, the most critical duty of SU is to sense the spectrum holes. In underlay access technique, the spectrum can be shared by PUs and SUs simultaneously. In addition, using harvested energy from RF signals for both transmitting data and sensing channel may significantly improve channel allocation performance. powering a cognitive radio network $(\mathrm{CRN})$ with RF energy can provide a both spectrum and energy-efficient solution for wireless networking.

This study not only tries to satisfy energy and collusion constraints, but also aims to minimize the average AoI when allocating channels to SUs which topic is recently having extreme importance. In the studied model, static primary and secondary pairs exist simultaneously and are scattered as independent homogeneous Poisson point processes (HPPPs) in a unit square area, as given in [53]. It is assumed that the coverage area of an PT is splitted into sub areas as overlay mode area, underlay mode area, guard zone, and harvesting zone (HZ). Guard zone is the area where the ST cannot utilize the busy channel of the PT, despite reducing the power level and its radius is defined as rg to protect its intended receiver from ST interference. The energy harvester in the ST should contain a power conversion circuit that can get DC power from the RF signals [53]. HZ is defined by the radius of $r_{h}$ where an ST can harvest from PT. Node locations are assumed to be fixed. A centralized spectrum server (controller) is supposed to exist in the CR network to which SUs report their positions, available channels, and current energy levels. It is assumed that this communication occurs in a band outside of the hired spectrum [56]. So, spectrum/energy management and flow routing are straightforward. 
In the model, each secondary user tells an access point (AP) which may be a base station or Internet of Things (IoT) sensor and assumed to be dressed with more than one wireless interface, such that each secondary user may use more than one channel. Besides the main function of communication with other APs, the AP also makes the SDN forwarding functionality, and also observes the traffic request from the related devices and the spectrum utilization. Each AP sends its gathered data to the controller, which is in charge of allocating channels according to the channel situations, transmission rates, energy and AoI levels. In addition to satisfying energy and collusion constraints, proposed m-channel allocation mechanism aims to minimize the average AoI by making effective sensing and update decisions over a finite horizon where harvested energy can be used for both data transmission and channel sensing.

It is supposed there are K PTs (so K available channels) to be used by the STs which is smaller than the number of STs (S), so there will be co-channel interference between users. The transmit power of $\mathrm{ST}_{i}$ is denoted by $\mathrm{P}_{i}$, and the average channel gain between the devices and $\mathrm{ST}_{j}$ in channel $\mathrm{l}$ is represented by $\mathrm{G}_{j}^{l}$ and the interference from the devices of $\mathrm{ST}_{j}$ to $\mathrm{ST}_{m}$ over channel 1 is shown by $\mathrm{I}_{j, m}^{l}$. So, the transmit rate $\mathrm{C}_{j}^{l}$ of $\mathrm{ST}_{j}$ over channel $\mathrm{l}$ can be defined as follows [52]:

$$
C_{j}^{l}=B \log \left(1+P_{j} G_{j}^{l} /\left(\sum_{j \neq m}^{S} I_{j, m}^{l}+\sigma^{2}\right)\right),
$$

,where B depicts the bandwidth of each channel in Hertz, and $\sigma^{2}$ is the noise power at the ST. As an ST is assumed to have m-channel transmission capability, the total transmit rate of an $\mathrm{ST}_{i}$ can be defined as:

$$
C_{i}=\sum_{m=1}^{K} C_{i}^{m}
$$

So, from these equations it is obvious that there is a trade off between increasing total transmit rate and co-channel interference. When a channel is utilized by more STs, more co-channel interference will appear and therefore the transmit rate will be restricted. Consequently, another measure of AoI may be used to represent the performance of channel allocation mechanism under this perspective.

\subsection{Age of Information Model}

The analysis can be simplified by representing transmission process of each ST as an $\mathrm{M} / \mathrm{G} / 1$ queueing system where it has total transmit rate $\mathrm{C}_{i}$ and traffic arrival rate is $\lambda_{i}$. As the delay analysis in the classical queuing model mainly depends on the mean queueing lag, it cannot fully represent the critical need on the information freshness in industrial applications, therefore, in this study, AoI is adopted as a measure of showing freshness of information [52].

If packets are assumed to be sent at time $\mathrm{t}_{1}, \mathrm{t}_{2}, \ldots, \mathrm{t}_{p}$ and obtained successfully at time $\mathrm{t}_{1}^{\prime}, \mathrm{t}_{2}^{\prime}, \ldots \mathrm{t}_{p}^{\prime}$, then AoI is calculated by $\mathrm{t}_{i^{-}}^{\prime} \mathrm{t}_{i-1}^{\prime}$ and the average AoI can be obtained as follows [52]:

$$
E\left[A_{i}\right]=\lim _{m \rightarrow \infty} 1 / m \sum_{i=1}^{m} t_{i}^{\prime}-t_{i-1}^{\prime}
$$


Utilizing the analysis for an $\mathrm{M} / \mathrm{G} / 1$ queueing system given in [57], the AoI can be given by the following equation

$$
E\left[A_{i}\right]=\frac{\lambda_{i} E\left[D^{2}\right] / C_{i}^{2}}{2 C_{i}\left(C_{i}-\lambda_{i} E[D]\right)}+\frac{E[D]}{C_{i}}+\frac{1+\lambda_{i} E[D] / C_{i}}{\lambda_{i} \Phi\left(-\frac{\lambda_{i}}{C_{i}}\right)}
$$

Here, $\mathrm{E}[\mathrm{D}]$ and $\mathrm{E}\left[D^{2}\right]$ are the first and second moments of the packet sizes. The packet size can be thought of to be a random variable following a general distribution and it can be shown by a moment generating function $\Phi(\mathrm{t})$.

ST utilizes its harvested and accumulated energy for both catching spectrum holes and doing transmission. ST can do harvesting, underlay or overlay transmission based on its location and how distant it is to the channel owner primary transmitter [53]. An ST should sense spectrum holes to avoid collisions with the primary pair [38]. An ST harvests energy of $\mathrm{EL}_{h}^{T}$ and spends it $\left(\mathrm{EL}_{c}^{T}\right)$ for selfish spectrum entry. Sensing and slot durations are assumed to be $\mathrm{t}$ and $\mathrm{T}$ seconds respectively. As the energy arrival process is random, ST behaves either actively or inactively depending on the energy level at the start of one time slot $\left(\mathrm{EL}_{t}\right)$. If this energy level is enough, it can sense spectrum and transmission or otherwise stays idle.

In this study, since not only the channel distribution performance and corresponding throughput is important, but also freshness of information is critical, it should be also taken into account during flow. On the other hand, it is also important to prioritize STs when allocating channels based on their initial energy levels and more importantly their harvesting capability as they play important role to utilize channels. Therefore, first STs are given priorities as below:

$$
\operatorname{Pri}_{S T}(i, j)=\text { Guard }_{S T(i, j)} *\left(0.4 * E L(i) / E L_{\max }+0.6 * \text { Harvest }_{S T(i, j)}\right),
$$

As long as initial energy levels are not high enough compared to harvested energy levels, which is a reasonable assumption in real life communication, harvesting capability plays more important role in being able to make transmission in next time slots, so given higher priority during prioritization. Therefore, from two nodes which are exactly in the same distance to a PT and having same initial energy level, the one with more harvesting capability will be higherly prioritized. Here,

- $\operatorname{Guard}_{S T}(\mathrm{i}, \mathrm{j})$ shows whether ST(i) is located in the guard zone of PT(j) (owner of channel j). 1 means located outside the guard zone and 0 otherwise as ST(i) can never transmit using channel j;

- EL(i) is the existing energy level of ST(i) which is given in joules, the maximum of all energy levels is given by $\mathrm{EL}_{\max }$ which means normalizing the energy component; and

- finally, Harvest ${ }_{S T}(\mathrm{i}, \mathrm{j})$ is 1 if $\mathrm{ST}(\mathrm{i})$ is located in the HZ of the PT(j), and 0 otherwise.

\subsection{Problem definition}

The proposed mechanisms not only aim to maximize channel distribution performance, but also to minimize age of information in a energy harvesting CRN under both collusion and energy constraints. The problem can be formulized as below: For all channels $\mathrm{k}=1, \ldots \mathrm{K}$, 
- Minimize average $\left(\mathrm{AoI}_{i}\right)$,

- Maximize channel distribution performance, $\forall \mathrm{ST}_{i}, \mathrm{i}=1, \ldots \mathrm{S}$ s.t.

Total interference $<$ acceptable total interference for a PR, for a given outage ratio,

$\lambda_{i}{ }^{*} \mathrm{D}<\mathrm{C}_{i} ; \forall \mathrm{i}$; (the traffic load for each $\mathrm{ST}$ should be lower than its overall service rate),

$\mathrm{EL}(\mathrm{i})>\mathrm{EL}_{s}+\mathrm{EL}_{\text {transmit }}, \forall \mathrm{i}$, where $\mathrm{EL}_{s}+\mathrm{EL}_{t}$ represent necessary energy levels for sensing spectrum and making transmission (overlay or underlay).

It can be simply seen that this problem is NP-hard and cannot be solved efficiently.

\section{Greedy and Proposed Algorithms}

In this study, a greedy mechanism is taken as a basis for optimizing age of information data while maximizing throughput to compare the proposed mechanisms and highlight its performance. The results of greedy and proposed mechanisms are compared and the aim of all these mechanisms is to minimize age of information data when maximizing multi-channel allocation performance in an energy harvesting CRN.

In this study, a greedy mechanism is taken as a basis for optimizing age of information data while maximizing throughput to compare the proposed mechanisms and highlight its performance. The results of greedy and proposed mechanisms are compared and the aim of all these mechanisms is to minimize age of information data when maximizing multi-channel allocation performance in an energy harvesting CRN.

\subsubsection{Greedy Algorithm}

Table 1 gives the input and output parameters and steps of the greedy allocation algorithm.

\subsubsection{Proposed Algorithm}

Unlike the allocation mechanism proposed in [53], the proposed method in the current study takes into account age of information -which is direct result of cochannel interference- as a measure to represent the freshness of data when allocating multi-channels to STs. Also, collusion and energy constraints are still considered to protect PTs from STs which are capable of energy harvesting. Depending on the mechanism of limiting average AoI at each iteration, there are 2 versions of the proposed mechanism. In the first version, an ST can obtain the new channel as long as the increase of AoI stays under a predefined amount of percentage, otherwise cannot obtain the channel. On the other hand, in the second version, an ST can utilize the channel if the new AoI stays below the average AoI of previous iteration, otherwise cannot get the new channel. The input and output parameters and steps of these two algorithms (version I and II) are given in Table 2 and Table 3 respectively. 
Table 1: Greedy Algorithm

\begin{tabular}{|c|c|}
\hline Input Parameters: & $\begin{array}{l}\text { - Interference graph G; } \\
\text { - Number of wireless interfaces of each secondary user; } \\
\text { - Number of primary users; } \\
\text { - Transmission power of primary and secondary users; } \\
\text { - Initial energy levels of ST users; } \\
\text { - Pefinitions of overlay, underlay, and guard zones; } \\
\text { Primary and secondary overlay/underlay transmission power levels; } \\
\text { - Slonsing power; } \\
\text { - Sensing period; } \\
\text { - Transmission period; } \\
\text { - Signal to interference plus noise ratio. } \\
\text { - Matrices of STs for overlay, underlay, harvesting and guard zones, respectively, } \\
\text { colum of which consists of values } 1 \text { or 0. Rows of matrices consist of STs, and } \\
\text { located in corresponding zone, otherwise 0. }\end{array}$ \\
\hline Output Parameter & $\begin{array}{l}\text { Average age of information } \\
\text { Channel distribution fairness index. }\end{array}$ \\
\hline Step 1: & $\begin{array}{l}\text { - Create interference matrices for each ST towards each PT for overlay and } \\
\text { underlay transmission modes, using overlay and underlay transmission powers } \\
\text { of each ST and distance between ST and PT [39]. }\end{array}$ \\
\hline Step 2: & $\begin{array}{l}\text { - Select the least used channel from available channels and calculate the pro- } \\
\text { posed metric given in Eq. (5) for each ST towards that channel, and sort the list } \\
\text { in descending order of metric. }\end{array}$ \\
\hline Step 3: & $\begin{array}{l}\text { - Sense if the channel found in step } 2 \text { is idle or busy. } \\
\text { If channel= =idle; then, } \\
\text { - proceed to step } 4 \text {. } \\
\text { else, } \\
\text { - proceed to step } 5 \text {. }\end{array}$ \\
\hline Step 4: & $\begin{array}{l}\text { - Find the ST with best metric - which is not checked yet for this channel- and } \\
\text { examine whether its energy suffices for overlay transmission using the channel } \\
\text { found in step } 2 \text {. } \\
\text { If the energy is sufficient and the consequent total interference value on the } \\
\text { owner PT of the channel does not exceed the necessary interference threshold } \\
\text { for a given SINR level and neighbour STs did not already get this channel, then, } \\
\text { - allocate the channel to the ST, } \\
\text { - recalculate the energy level of ST, } \\
\text { - subtract sensing and overlay transmission energies from the initial energy, } \\
\text { - revise the lists of used channels of affected nodes. } \\
\text { - remove the nodes from main interference graph which fulfilled all their } \\
\text { channel requirements and continue with step } 6 \text {. }\end{array}$ \\
\hline Step 5: & $\begin{array}{l}\text { - Find the ST with best metric -which is not checked yet- and examine whether } \\
\text { it is located outside the interference range of the channel owner. } \\
\text { If yes; then, } \\
\text { - check if energy level of the node is enough for overlay transmission, cor- } \\
\text { responding total interference value on the channel owner PT satisfies required } \\
\text { SINR ratio and neighbouring STs did not get this channel yet, } \\
\text { If yes, then, } \\
\text { allocate the channel to the ST, } \\
\text { - recompute the energy level of ST, } \\
\text { subtract sensing and overlay transmission energies from the existing } \\
\text { energy level, } \\
\text { - revise the lists of used channels of affected nodes, } \\
\text { remove the nodes from main interference graph which fulfilled all their } \\
\text { channel requirements and continue with step } 6 \text {. }\end{array}$ \\
\hline Step 6: & $\begin{array}{l}\text { Examine if all STs are tested towards the selected channel. } \\
\text { If yes, then, } \\
\text { - continue with step } 2 \text { by selecting another available channel. If all channels } \\
\text { have already been tested, proceed to step } 7 \text {. } \\
\text { else, } \\
\text { - return to step } 3 .\end{array}$ \\
\hline Step 7: & $\begin{array}{l}\text { - Check if the number of nodes in the main interference graph is greater than } 1 . \\
\text { If yes, then, } \\
\text { check if no more channel can be assigned due to energy insufficiency. } \\
\text { If yes, then, } \\
\text { - calculate average age of information values for all STs using Equa- } \\
\text { tion (4) and finish. } \\
\text { else, } \\
\text { - return to step } 1 \text { and restart channel allocation mechanism with new } \\
\text { updated energy levels. } \\
\text { else, } \\
\text { - then calculate average age of information values for all STs using Equa- } \\
\text { tion (4) and finish. }\end{array}$ \\
\hline
\end{tabular}


Table 2: Proposed algorithm.VersionI)

\begin{tabular}{|c|c|}
\hline Input Parameters: & $\begin{array}{l}\text { - Interference graph G; } \\
\text { - Number of wireless interfaces of each secondary user; } \\
\text { - Number of primary users; } \\
\text { - Transmission power of primary and secondary users; } \\
\text { - Definititions of overlay, underlay, and guard zones; } \\
\text { - Primary and secondary overlay/underlay transmission power levels; } \\
\text { - Sensing power; } \\
\text { - Slot duration; } \\
\text { - Transing period; } \\
\text { - Signal to interferionce plus noise ratio. } \\
\text { - Matrices of STs for overlay, underlay, harvesting and guard zones, respectively, } \\
\text { each of which consists of values } 1 \text { or 0. Rows of matrices consist of STs, and } \\
\text { columns correspond to channels. Value of matrix element is set to } 1 \text { if ST is } \\
\text { located in corresponding zone, otherwise 0. }\end{array}$ \\
\hline Output Parameter & $\begin{array}{l}\text { Average age of information } \\
\text { Channel distribution fairness index. }\end{array}$ \\
\hline Step 1: & $\begin{array}{l}\text { - Create interference matrices for each ST towards each PT for overlay and } \\
\text { underlay transmission modes, using overlay and underlay transmission powers } \\
\text { of each ST and distance between ST and PT [39]. }\end{array}$ \\
\hline Step 2: & $\begin{array}{l}\text { - Select the least used channel from available channels and calculate the pro- } \\
\text { posed metric given in Eq. (5) for each ST towards that channel, and sort the list } \\
\text { in descending order of metric. }\end{array}$ \\
\hline Step 3: & $\begin{array}{l}\text { - Sense if the channel found in step } 2 \text { is idle or busy. } \\
\text { If channel==idle; then, } \\
\text { - proceed to step } 4 \text {. } \\
\text { else, } \\
\text { - proceed to step } 5 \text {. }\end{array}$ \\
\hline Step 4 & $\begin{array}{l}\text { Find the ST with best metric -which is not checked yet- and examine whether } \\
\text { its energy is sufficient for overlay transmission using the channel found in step } \\
\text { 1. - If the energy level of ST is sufficient and the resulting total interference } \\
\text { value on the PT owning the channel does not exceed the necessary interference } \\
\text { threshold for a given SINR level and neighbour STs did not already get this } \\
\text { channel, then, } \\
\text { - allocate the channel to the ST, } \\
\text { - recalculate the energy level of ST, } \\
\text { - revtract sensing and overlay transmission energies from the initial energy, } \\
\text { - remove the nodes from main interference graph which fulfilled all their } \\
\text { channel requirements and continue with step } 6 \text {. }\end{array}$ \\
\hline Step 5: & 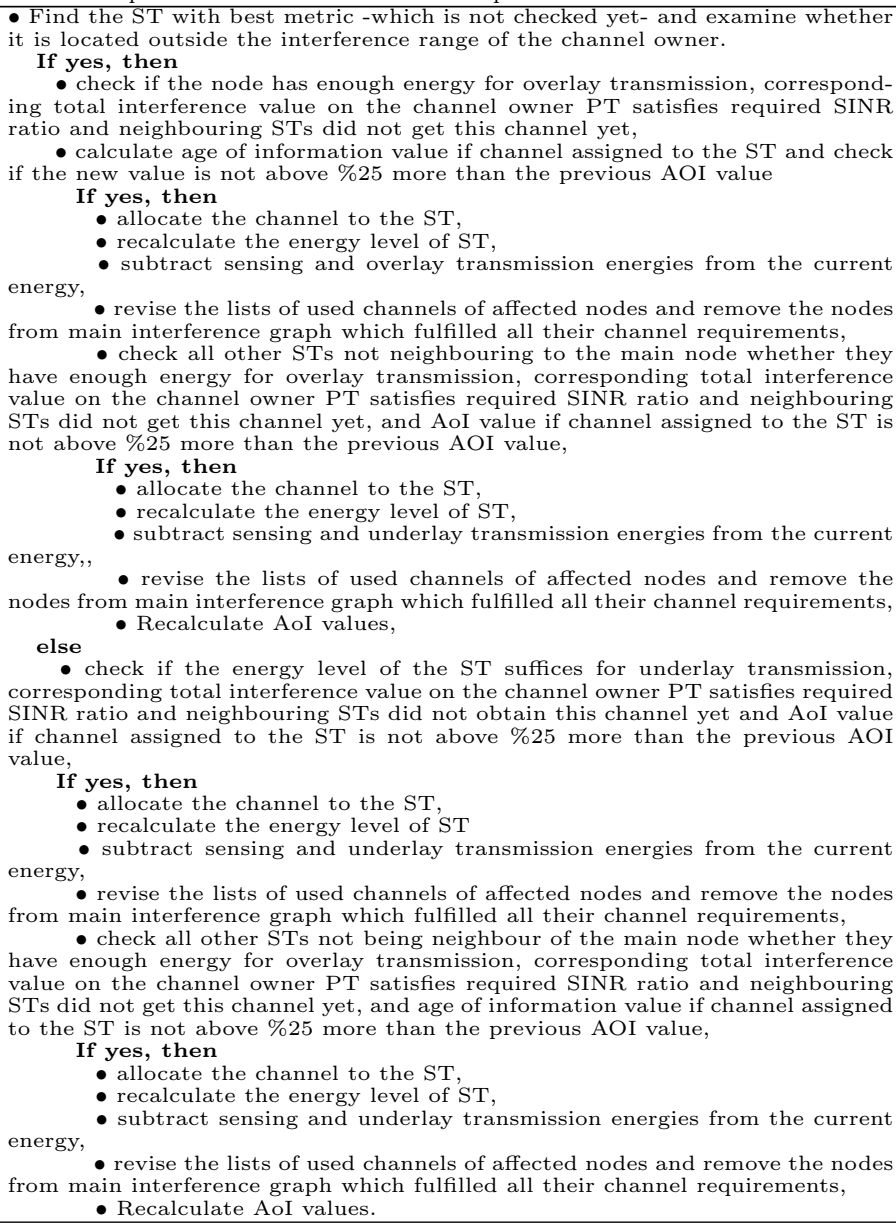 \\
\hline
\end{tabular}


Table 3: Proposed algorithm.VersionII)

\begin{tabular}{|c|c|}
\hline Input Parameters: & $\begin{array}{l}\text { - Interference graph G; } \\
\text { - Number of wireless interfaces of each secondary user; } \\
\text { - Number of primary users; } \\
\text { Transmission power of primary and secondary users; } \\
\text { - Initial energy levels of ST users; } \\
\text { - Definitions of overlay, underlay, and guard zones; } \\
\text { Primary and secondary overlay/underlay transmission power levels; } \\
\text { - Sensing power; } \\
\text { - Sensing period; } \\
\text { - Transmission period; } \\
\text { - Signal to interference plus noise ratio. } \\
\text { - Matrices of STs for overlay, underlay, harvesting and guard zones, respectively, } \\
\text { colum of which consists of values } 1 \text { or 0. Rows of matrices consist of STs, and } \\
\text { located in correspond to channels. Value of matrix element is set to } 1 \text { if ST is } \\
\end{array}$ \\
\hline Output Parameter & $\begin{array}{l}\text { Average age of information } \\
\text { Channel distribution fairness index. }\end{array}$ \\
\hline Step 1: & $\begin{array}{l}\text { - Create interference matrices for each ST towards each PT for overlay and } \\
\text { underlay transmission modes, using overlay and underlay transmission powers } \\
\text { of each ST and distance between ST and PT [39]. Calculate average AoI value. }\end{array}$ \\
\hline Step 2: & $\begin{array}{l}\text { - Select the least used channel from available channels and calculate the pro- } \\
\text { posed metric given in Eq. (5) for each ST towards that channel, and sort the list } \\
\text { in descending order of metric. }\end{array}$ \\
\hline Step 3: & $\begin{array}{l}\text { Sense if the channel found in step } 2 \text { is idle or busy. } \\
\text { If channel==idle; then, } \\
\text { - proceed to step } 4 \text {. } \\
\text { else, } \\
\text { - proceed to step } 5 \text {. }\end{array}$ \\
\hline Step 4 & $\begin{array}{l}\text { Find the ST with best metric -which is not checked yet- and examine whether } \\
\text { its energy is sufficient for overlay transmission using the channel found in step } \\
\text { 1. If the energy level of ST is sufficient and the resulting total interference } \\
\text { value on the PT owning the channel does not exceed the necessary interference } \\
\text { threshold for a given SINR level and neighbour STs did not already get this } \\
\text { channel, then, } \\
\text { - allocate the channel to the ST, } \\
\text { recalculate the energy level of ST, } \\
\text { - subtract sensing and overlay transmission energies from the initial energy, } \\
\text { revise the lists of used channels of affected nodes, } \\
\text { chanove the nodes from main interference graph which fulfilled all their } \\
\text { channel requirements and continue with step } 6 \text {. }\end{array}$ \\
\hline Step 5: & 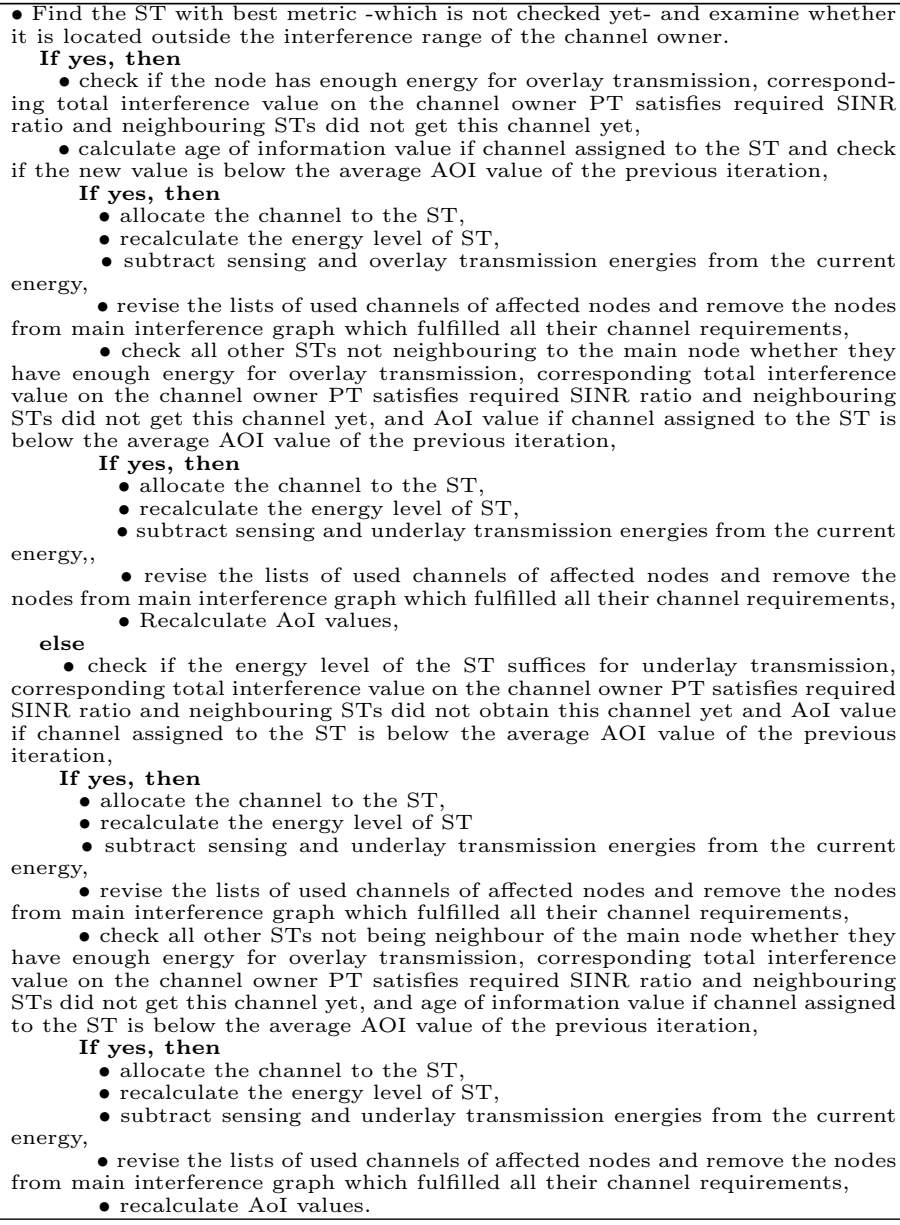 \\
\hline
\end{tabular}




\section{Results and Discussion}

In this section, simulation results to evaluate the performance of the proposed schemes are presented. The system parameter definitions and values used in simulations are given in Table 4 [13], [25], [54]. Packet sizes are assumed to be exponentially distributed with mean of $5 \mathrm{Kbits}$ and mean value of poisson distributed traffic arrival rate is 500 packets/slot. Depending on whether ST is located in the guard zone, harvesting zone, underlay or overlay regions of a channel owner PT and current energy level, it either senses channel, or makes overlay/underlay transmission or stays idle [53]. To prove the performance and superiority of the proposed schemes in terms of AoI, both average AoI and channel distribution performance are compared. To represent AoI, Equation (4) is computed at every channel assigment to each ST and at the end of each iteraton all values are averaged for a given signal plus interference to noise ratio (SINR) such that collusion and energy constraints are satisfied. For interference and SINR calculations, equations given in [53] are used. Also, channel distribution performance is calculated using equation given in [53].

In order to prove performance of proposed schemes, 10000 independent iterations were run and all experimental results were averaged. PUs and SUs are independently HPPP based distributed in a $(1000 \times 1000) \mathrm{m}^{2}$ area. The number of all available channel is equal to the number of PTs. Each SU may represent either a base station or Internet of Things (IoT) sensor and they are assumed to have more than one wireless interface such that transmitting over more than one channel at the same time. During the simulations a typical path loss of value 3.7 was used. All simulations were run with MATLAB (FIGES A.S, Istanbul, Turkey).

Table 4: Parameters Used In Simulations.

\begin{tabular}{|l|l|l|}
\hline Symbol & Description & Value \\
\hline $\mathrm{P}_{S}$ & Power Level for Sensing & $110 \mathrm{~mW}$ \\
\hline $\mathrm{P}_{O}$ & Power Level for Overlay Transmission & $100 \mathrm{~mW}$ \\
\hline $\mathrm{P}_{U}$ & Power Level for Underlay Transmission & $60 \mathrm{~mW}$ \\
\hline $\mathrm{T}$ & Duration of each Slot & $1 \mathrm{~ms}$ \\
\hline$\tau$ & Duration of each Sensing Period & $0.002 \mathrm{~ms}$ \\
\hline $\mathrm{T}-\tau$ & Duration of each Transmission Period & $0.098 \mathrm{~ms}$ \\
\hline $\mathrm{E}$ & Initial Energy Level & $\begin{array}{l}\text { random value in range } \\
{[0, \max (\mathrm{E})]}\end{array}$ \\
\hline $\mathrm{SINR}$ & Signal to Interference Plus Noise Ratio & $\mathrm{dB}$ \\
\hline $\mathrm{EL}_{\max }$ & Maximum of All Energy Levels & $\mathrm{mJ}$ \\
\hline $\mathrm{EL}_{i}$ & Existing Energy Level & $\mathrm{mJ}$ \\
\hline
\end{tabular}




\section{Complexity Analysis of Algorithms:}

In this section, the complexity of both proposed and greedy algorithms are analysed. The assumption is that there are $\mathrm{S}$ secondary and $\mathrm{K}$ primary transmitters and the number of iterations executed is $\mathrm{N}$.

\section{The greedy algorithm consists of the following phases:}

- In the first level, interference matrix and energy level computations have complexity of $\mathrm{O}\left(\mathrm{N}^{*} \mathrm{~S}\right)$.

- In the second level, channel selection and node metric computations $\mathrm{O}(\mathrm{K})$, node selection with highest metric $(\mathrm{O}(\mathrm{S}))$, interference calculations for all STs obtained this channel, channel allocation and recalculation of energy levels which will be repeated for all elements of interference graph have complexity of $\mathrm{O}\left(\mathrm{N}^{*}\left(5^{*} \mathrm{~K}^{*} \mathrm{~S} * \mathrm{~S}+(\mathrm{S}+\mathrm{K})\right)\right.$.

- In the last step, average AoI calculations $\mathrm{O}\left(\mathrm{S}^{*} \mathrm{~S}\right)$, checking if all nodes are tested and any more allocations can be applied requires complexity of $\mathrm{O}(\mathrm{S})$.

So, the overall computational complexity of the greedy algorithm is $\mathbf{O}\left(\mathbf{N}^{*}\left(\mathbf{5}^{*} \mathbf{K}^{*} \mathbf{S}^{*} \mathbf{S}\right.\right.$ $\left.\left.+(\mathbf{S}+\mathbf{K})+\mathbf{S}^{*} \mathbf{S}\right)\right)$

\section{The proposed algorithm consists of the following phases:}

- In the first level, interference matrix and energy level computations have complexity of $\mathrm{O}\left(\mathrm{N}^{*} \mathrm{~S}\right)$.

- In the second phase, channel selection and node metric calculations $\mathrm{O}(\mathrm{K})$, node selection with highest metric $(\mathrm{O}(\mathrm{S}))$, computation of interference for the selected channel, channel allocation, average AoI and energy level calculations $\left(\mathrm{O}\left(5^{*} \mathrm{~K}^{*} \mathrm{~S} * \mathrm{~S}+(\mathrm{K}+\mathrm{S})\right)\right)$ which will be reiterated for all elements of the graph $(\mathrm{O}(\mathrm{S}))$. So, it requires complexity of $\mathrm{O}\left(\mathrm{N}^{*}\left(5^{*} \mathrm{~K}^{*} \mathrm{~S} * \mathrm{~S} * \mathrm{~S}+(\mathrm{S}+\mathrm{K})\right)\right.$.

- In the last step, checking if all nodes are tested and any more allocations can be applied requires complexity of $\mathrm{O}(\mathrm{S})$.

As a result, the overall computational complexity of the proposed algorithm is $\mathbf{O}\left(\mathbf{N}^{*}\left(5^{*} \mathbf{S}^{3 *} \mathbf{K}+(\mathbf{K}+\mathbf{S})\right)\right)$. Compared with greedy algorithm, the proposed algorithm has higher complexity because of average AoI computations for each member of interference graph at each iteration. 
To prove superiority of proposed algorithms, 5 different experiments were run and the results are as follows:

Experiment 1. In the first experiment, average AoI calculated according to Equation (4) and the channel distribution performance (ChDistFairness) calculated using equation used in [53] for various SINR (outage) threshold values are examined. Parameters used in this experiment can be summarized as:

- There are 4 PTs which means the number of available channels is 2 and the number of idle channels varies randomly between 0 and 3 .

- The Poisson distribution density of STs was about 0.0003 in the $250 * 250$ $\mathrm{m}^{2}$ area around the PT.

- Average estimated percentage of STs in HZ is about 2.9\%, calculated according to Equation drived in [54].

\section{Results can be commented on as below:}

- Average AoI values are investigated within increasing time slots for varying SINR (outage) threshold values. The criterion used for controlling AoI values at each iteration is $50 \%$ increase, which implies if the assignment of the channel to an ST will result in an increment in AoI level higher than 50\%, ST will not be assigned that channel, otherwise will able to utilize the channel. As the Figure 1 depicts, average AoI values fall within increasing SINR (outage) thresholds as anticipated since channel distribution is restricted with increase of SINR, thereby decreasing cochannel interference and AoI drops as well.

- On the other hand, different colors represent results for differing timeslots starting from slot 1 to 5 . Since multi channels are assigned to STs in coming time slots -via contribution of energy harvesting-, cochannel interference increases and therefore AoI values for the same SINR (outage) threshold rise as well.

- Channel allocation metrics calculated according to equation used in [53] is also investigated for different SINR (outage) thresholds. As Figure 2 shows channel distribution metric results drop within coming time slots by means of energy harvesting. On the other hand, because of AoI restriction taken into account during each allocation, allocation performance decreases for increasing SINR (outage) threshold values, so allocation metric values get higher.

Experiment 2. In the second experiment, performance of proposed algorithm and greedy mechanism are compared in terms of AoI values. Average AoI computed according to Equation (4) and channel allocation performance metric (ChDistFairness) obtained according to equation given in [53] for various SINR (outage) threshold values are examined.

Parameters used in this experiment can be summarized as:

- There are 4 PTs which means the number of available channels is 2 and the number of idle channels varies randomly between 0 and 2 .

- The Poisson distribution density of STs was about 0.0003 in the $250 * 250$ $\mathrm{m}^{2}$ area around the PT.

- Average estimated percentage of STs in HZ is about 2.9\%, calculated according to Equation drived in [54].

Results can be on commented as below: 
- As it is seen in the Figure 3, average AoI of proposed mechanism is much smaller than those of greedy mechanism where hybrid allocation is based only on sorting STs by their degrees in the interference graph, where they can still do energy harvesting. This shows superiority of the proposed algorithm.

- In addition, in terms of channel distribution performance, proposed algorithm does much better than the greedy one. Results are depicted in Figure 4 and this proves proposed algorithm performs much better in terms of both average AoI and channel distribution. When allocation performance is improved, AoI performance is supposed to drop as a result of increasing co-channel interference. Even though the proposed scheme considers average AoI values and puts restriction on channel assignment accordingly, both average AoI and allocation performance are still outstandingly improved than the greedy algorithm.

Experiment 3. In this experiment, density of STs around PTs increased from 0.0003 in the $250 \times 250 \mathrm{~m}^{2}$ to 0.001 in $150 \times 150 \mathrm{~m}^{2}$ area and percentage of harvesting STs changed from $3 \%$ to $12 \%$.

Parameters used in this experiment can be summarized as:

- There are 4 PTs meaning the number of available channels is 2 and the number of idle channels varies randomly between 0 and 2 .

- The Poisson distribution density of STs was about 0.001 in the $150 \times 150 \mathrm{~m}^{2}$ area around the PT.

- Average estimated percentage of STs in HZ is about $12 \%$, calculated according to Equation drived in [54].

\section{Results can be commented on as below:}

- As STs in the harvesting zones get denser, channel allocation performance gets better at lower SINR (outage) threshold values because of higher number of harvesting STs, especially till about $20 \mathrm{~dB}$. This is clearly seen in the Figure 5. Afterwards, however, interference constraint gets more dominant and hence channel distribution performance of higher density STs gets worse.

- On the contrary, as a consequence of allocation of more channels, co-channel interference accordingly gets higher which causes higher average AoI values computed to Equation (4). This is seen in the Figure 6. Similarly, when percentage of STs in HZ is higher, AOI values get smaller after 20dB SINR (outage) threshold as co-channel interference drops.

Experiment 4. Number of STs and PTs increased from 17/4 to 30/8 respectively. Parameters used in this experiment are same as before, except for number of STs and PTs modified as above:

- There are 8 PTs which means the number of available channels is 4 and the number of idle channels varies randomly between 0 and 4 .

- The Poisson distribution density of STs was about 0.002 in the $400 * 400 \mathrm{~m}^{2}$ area around the PT.

- Average estimated percentage of STs in $\mathrm{HZ}$ is about 3\%, calculated according to Equation drived in [54].

\section{Results can be commented on as below:}


- As the number of PTs increased from 4 to 8, number of available channels also raise from 2 to 4 , keeping the percentage of harvesting STs at $3 \%$ for both. Hence, number of channels to be allocated gets higher which results in an increase in co-channel interference. As a result, average AOI values raise according to Equation (4).

- At the higher levels of SINR (outage) threshold, restriction on allocation of channels becomes more apperant, therefore co-channel interference drops accordingly.

- In addition, channel distribution performance of $30 \mathrm{STs}$ at $30 \mathrm{~dB}$ is much better than that of $17 \mathrm{STs}$ since more number of channels are available per ST. Accordingly, average AOI value at $30 \mathrm{~dB}$ of $30 \mathrm{STs}$ (blue line) is much smaller than that of 17 STs (red line). These results are seen in Figure 7 and Figure 8 respectively.

Experiment 5. In the fifth experiment, transmit power of overlay and underlay modes increased.

Parameters used in this experiment can be summarized as:

- There are 4 PTs which means the number of available channels is 2 and the number of idle channels varies randomly between 0 and 2 .

- The Poisson distribution density of STs is about 0.0008 in the $100 * 100 \mathrm{~m}^{2}$ area around the PT.

- Average estimated percentage of STs in HZ is about $3 \%$, calculated according to Equation drived in [54].

- Overlay and underlay transmission powers of STs increased from 60 to $100 \mathrm{~mW}$ and from 30 to $60 \mathrm{~mW}$ respectively.

\section{Results can be commented on as below:}

- In this experiment, overlay and underlay transmit powers are increased from $60 / 30 \mathrm{~mW}$ to $100 / 60 \mathrm{~mW}$ levels. However, because of increase in the interference levels on PTs accordingly, channel allocation performance decreases, as depicted by the Figure 9 and average AOI values drop as well even though co-channel interference increases as a result of transmit power raise, as seen in the Figure 10. This once more proves the advantage of the proposed mechanism.

Experiment 6. In the last experiment, criterion for AoI control altered and corresponding AoI and channel distribution performance results compared.

Parameters used in this experiment are same as previous one:

- There are 4 PTs which means the number of available channels is 2 and the number of idle channels varies randomly between 0 and 2 .

- The Poisson distribution density of STs is about 0.0008 in the $100 * 100 \mathrm{~m}^{2}$ area around the PT.

- Average estimated percentage of STs in HZ is about $3 \%$, calculated according to Equation drived in [54].

- Overlay and underlay transmission powers of STs are again $60 \mathrm{~mW}$ and $30 \mathrm{~mW}$ respectively.

- Criterion for AoI control changed as below: - Channel distribution is allowed if new AoI value for the ST will be under the average AoI of previous iteration, - Channel distribution is allowed if new AoI value for the ST will be not more 
than 1.5 times of the previous AoI value, - Channel distribution is allowed if new AoI value for the ST will be not more than 10 times of the previous AoI value..

\section{Results can be commented on as below:}

- In this experiment, criterion for AoI control at each channel assignment are modified. Average AoI values are compared and results are shown in Figure 11. As seen from the figure, average AoI values are the lowest when new AoI value of an ST is limited such that it will not exceed the previous average AoI vaue of previous iteration (green line). This is true for all SINR (outage) threshold values. As the AoI criterion changed such that channel assignment is not allowed when AoI increase will be more than 1.5 times of previous AoI value of the ST (blue line), average AoI values get a little higher, but lower than the values with the other criterion, that means, channel assignment is not allowed if AoI increase will be more than 10 times of previous AoI value of the ST (red line). This is because more AoI increase is permitted at each iteration, average AoI will be higher. However, if corresponding channel distribution performances are compared, the resulting values at the end of 7 iterations of all methods are same which is around 0.0504, as seen in the Figure 12. As a consequence, when limiting AoI such that new value will not exceed the average AoI of previous iteration, the performance is best in terms of both average AoI and channel allocation.

These results prove proposed algorithms show good performance in terms of AoI while protecting PUs under energy and collusion constraints.. Unlike previous studies, like [50] and [51], this paper studies both throughput maximization and AoI minimization for optimal m-channel allocation in hybrid CRNs with energy harvesting capability under both energy and collision constraints at the same time and this is the first study considering all these in a hybrid CRN. Consequently, all these simulation results prove, the proposed algorithms perform allocation with minimal AoI value and maximum channel allocation performance without disturbing channel owner PUs. This proves the superiority of the proposed mechanism.

\section{Conclusions}

In this study, the problem of average AOI minimization in allocation of multichannels to secondary users with ability of energy harvesting in a hybrid cognitive radio network is examined. SINR constraint on primary users are also taken into account such that they will not be affected by STs. Novel algorithms are proposed when channel allocation performance is maximized, average AOI values are minimized during allocation of multiple channels in a hybrid CRN in which STs are also capable of energy harvesting from RF signals. To minimize AOI values, initially, STs are sorted based on a proposed metric which considers both energy levels of STs and whether they can do harvesting or not. Having more energy and harvesting capability will have higher priority. Secondly, when allocating channels, AOI value of each ST for the channel is calculated and if the value stays below certain levels, it will have the chance to obtain the channel. AOI values are calculated based on cochannel interference between STs which strictly depends on their transmission rates and power levels and threshold level of AOI is defined based on different proposed criteria. 
To the best of author' s knowledge, this is the first study of AOI minimization during allocation of multi-channels to SUs with harvesting ability in a hybrid CRN while trying to maximize channel allocation performance under collusion and energy constraints. Either of overlay/underlay transmissions is selected according to the distance between ST and channel owner PT. Also, STs only existing in the harvesting zone can do harvesting and they are presumed to be equipped with the same number of harvesting equipments and wireless interfaces as the required number of channels such that harvesting from different channels at the same time becomes possible. Co-channel interference is taken into account in order to represent AoI values according to the Equation (2) and Equation (4). In order to compare and show superiority of the proposed mechanism, a greedy scheme without AoI control is utilized which does allocation of multi-channels based on the degrees of STs who can do energy harvesting in hybrid CRNs. The simulation results depict that restriction and control of AoI values based on proposed criteria in the proposed mechanism significantly reduce average AoI values while maximizing channel distribution performance without disturbing PU transmission. Also, as percentage of nodes in HZ increase, those STs in the coming time slots will have more chance to utilize channels thanks to having more energy, however, co-channel interference will be more affecting, thereby increasing average AoI values.

\section{References}

1. Kaul, S., Gruteser, M., Rai, V., \& Kenney, J. (2011a). Minimizing age of information in vehicular networks. In: 8th Annual IEEE Communications Society Conference on Sensor, Mesh and Ad Hoc Communications and Networks (SECON), 350-358.

2. Kaul, S., Yates, R. D., \& Gruteser, M. (2011b). On Piggybacking in Vehicular Networks. In: IEEE Global Telecommunications Conference (GLOBECOM), 1-5.

3. Kaul, S., Yates, R. D., \& Gruteser, M. (2012b.). Real-time status: How often should one update? In: IEEE International Conference on Computer Communications (INFOCOM), 2731-2735.

4. Yates, R. D., \& Kaul., S. (2012). Real-time status updating: Multiple sources. In: IEEE International Symposium on Information Theory (ISIT), 2666-2670.

5. Papadimitratos, P., Fortelle, A. D. L., Evenssen, K., Brignolo, R., \& Cosenza, S. (2009). Vehicular communication systems: En abling technologies, applications, and future outlook on intelligent transportation. IEEE Communications Magazine, 47(11), 84-95.

6. He, Q., Dán, G., \& Fodor, V. (2018). Minimizing age of correlated information for wireless camera networks. In Proc. IEEE International Conference on Computer Communications (INFO COM), 547-552.

7. He, Q., Dán, G., \& Fodor, V. (2019). Joint assignment and scheduling for minimizing age of correlated information. IEEE ACM Transactions on Networking, 27(5), 1887-1900.

8. Kadota, I., Sinha, A., Uysal-Biyikoglu, E., \& Singh, R., \& Modiano, E. (2018). Scheduling Policies for Minimizing Age of Information in Broadcast Wireless Networks. IEEE ACM Transactions on Networking, $6(6)$

9. Ko, J., Lu, C., Srivastava, M. B., Stankovic, J. A., Terzis, A., \& Welsh, M. (2010). Wireless sensor networks for healthcare. Proceedings of the IEEE, 98(11), 1947-1960.

10. Corke, P., Wark, T., Jurdak, R., Hu, W., Valencia, P., \& Moore, D. (2010). Environmental wireless sensor networks. Proceedings of the IEEE, 98(11), 1903-1917.

11. Karakasidis, A., Vassiliadis, P., \& Pitoura, E. (2005). Etl queues for active data warehousing. In Proc. of the 2nd international workshop on Information quality in information systems, ACM28-39. 37

12. Yu, H., Breslau, L., \& Shenker, S. (1999). A scalable web cache consistency architecture. ACM SIGCOMM Computer Communication Review, 29(4), 163-174.

13. Kam, C, Kompella, S., Nguyen, G. D., Wieselthier, J. E., \& Ephremides, A. (2017). Information freshness and popularity in mobile caching. in Proc. IEEE International Symposium on In formation Theory (ISIT), 136-140.

14. Yates, R. D., Ciblat, P., Yener, A., \& Wigger, M. (2017). Age-optimal constrained cache updating. In Proc. IEEE International Sym posium on Information Theory (ISIT), 141-145.

15. Zhong, J. ,Yates, R. D., \& Soljanin, E. (2018). Two freshness metrics for local cache refresh. in Proc. IEEE International Symposium on Information Theory (ISIT), 1924-1928.

16. Xiong, M. \& Ramamritham, K. (2004). Deriving deadlines and periods for real-time update transactions. IEEE Transactions on Computers, 53(5), 567-583.

17. Hu, Y.-C., \& Johnson, D. B. Ensuring cache freshness in on demand ad hoc network routing protocols. In Proc. ACM in ternational workshop on principles of mobile computing. ACM, 25-30. 
18. Kam, C., Kompella, S., Nguyen, G. D., Wieselthier, J. E., \& Ephremides, A. (2017). Modelling the age of information in emulated ad hoc networks. In Proc. IEEE Military Communications Conference $(M I L C O M), 436-441$.

19. Abd-Elmagid, M. A., \& Dhillon, H. S. (2018). Average peak age of-information minimization in UAV-assisted IoT networks. IEEE Transactions on Vehicular Technology, 68(2), 2003-2008.

20. Ekpenyong, A. E., \& Huang, Y. f. (2007). Feedback constraints for adaptive transmission. IEEE Signal Processing Magazine, 24(3), 69-78.

21. Ying, L., \& Shakkottai, S. (2011). On throughput optimality with delayed network-state information. IEEE Transactions on In formation Theory, 57(8), 5116-5132.

22. Kosta, A., Pappas, N., \& Angelakis, V. (2017). Age of information: A new concept, metric, and tool. Foundations and Trends in Networking, 12(3), 162-259.

23. Barakat, B., Yassine, H., Keates, S., Wassell, I., \& Arshad, K. 2019). How to Measure the Average and Peak Age of Information in Real Networks?. European Wireless 2019, 25th European Wireless Conference, Aarhus, Denmark, 1-5.

24. Kosta, A., Pappas, N., Ephremides, A., \& Angelakis, V. (2017). Age and value of information: Non-linear age case. In Proc. IEEE International Symposium on Information Theory (ISIT), 326-330.

25. Kosta, A., Pappas, N., Ephremides, A., \& Angelakis, V. (2020). The cost of delay in status updates and their value: Non-linear ageing. IEEE Transactions on Communications.

26. Kaul, S., Yates, R. D., \& Gruteser, M. (2012a). Status updates through queues. In: 46th Annual Conference on Information Sciences and Systems (CISS). 1-6.

27. Sun, Y., Uysal-Biyikoglu, E., Yates, R., Koksal, C. E., \& Shroff, N. B. (2016). Update or wait: How to keep your data fresh. In Proc. IEEE International Conference on Computer Communications (INFOCOM), 1-9.

28. Ayan, O., Vilgelm, M., Klügel, M., Hirche, S., \& Kellerer, W. (2019). Age-of-information vs. value-of-information scheduling for cel lular networked control systems. In Proc. ACM/IEEE Inter national Conference on Cyber-Physical Systems, 109-117.

29. Tang, H. , Wang, J., Tang, Z., \& Song, J. (2019). Scheduling to minimize age of synchronization in wireless broadcast networks with random updates. In Proc. IEEE International Symposium on Information Theory (ISIT), 1027-1031.

30. Maatouk, A., Kriouile, S., Assaad, M., \& Ephremides, A. (2019). The age of incorrect information: A new performance metric for status updates.[Online]. Available: https: //arxiv.org/abs/1907.06604.

31. Kaul, S., Gruteser, M., Rai, V., \& Kenney, J. (2011). Minimizing age of information in vehicular networks. In Proc. 8th Annual IEEE Communications Society Conference on Sensor, Mesh and Ad Hoc Communications and Networks (SECON), 350-358.

32. Kaul, S., Yates, R., \& Gruteser, M. (2012). Real-time status: How often should one update?.In Proc. IEEE INFOCOM, 2731-2735.

33. Yates, R. D. (2015). Lazy is timely: Status updates by an energy harvesting source. In Proc. IEEE Intern. Sympos. on Inf. Theory, 3008-3012.

34. Bacinoglu, B. T., Ceran, E. T., \& Uysal-Biyikoglu, E. (2015). Age of information under energy replenishment constraints. In Proc. IEEE Inf. Theory and Application Workshop, 25-31.

35. $\mathrm{Wu}, \mathrm{X}$., Yang, J., \& Wu, J. (2018). Optimal status update for age of information minimization with an energy harvesting source. IEEE Trans. Green Commun. and Networking, 2(1), 193-204.

36. Arafa, A., Yang, J., Ulukus, S. \& Poor, H. V. (2018). Age-minimal transmission for energy harvesting sensors with finite batteries. Online policies. arXiv:1806.07271.

37. Arafa, A., \& Ulukus, S. (2017). Age-minimal transmission in energy harvesting two-hop networks. In Proc. IEEE Glob. Commun. Conf., Singapore, 1-6.

38. Wu, X., Yang, J., \& Wu, J. (2018). Optimal status update for age of information minimization with an energy harvesting source. IEEE Trans. Green Commun. Netw., 2(1), 193-204.

39. Arafa, A.,Yang, J., \& Ulukus, S. (2018). Age-minimal online policies for energy harvesting sensors with random battery recharges. in Proc. IEEE Int. Conf. Commun, 1-6.

40. Arafa, A., Yang, J., Ulukus, S., \& Poor, H. V. (2020). Age-minimal transmission for energy harvesting sensors with finite batteries: Online policies. IEEE Transactions on Information Theory, 66(1).

41. Menon, R., Buehrer, R. M., \& Reed, J. H. (2005). Outage probability based comparison of underlay and overlay spectrum sharing techniques. In Proc. First IEEE International Symposium on New Frontiers in Dynamic Spectrum Access Networks (DySPAN), 101-109.

42. Xu, W., Zhang, J., Zhang, P., \& Tellambura, C. (2012). Outage probability of decode-and-forward cognitive relay in presence of primary user's interference. IEEE Communications Letters, 16(8), 1252- 1255.

43. Lee, J., Wang, H., Andrews, J. G., \& Hong, D. (2011). Outage probability of cognitive relay networks with interference constraints. IEEE Transactions on Wireless Communications, 10(2), 390-395.

44. Khoshkholgh, M. G., Navaie, K., \& Yanikomeroglu, H. (2010). Access strategies for spectrum sharing in fading environment: Overlay, underlay, and +mixed. IEEE Transactions on Mobile Computing, 9(12), 1780-1793.

45. Gu, Y., Chen, H., Zhai, C., Li, Y., \& Vucetic, B. (2019). Minimizing Age of Information in Cognitive Radio-based loT Systems: Underlay or Overlay?. IEEE Internet of Things Journal, 6(6), 10273 - 10288

46. Sultan, A. (2012). Sensing and transmit energy optimization for an energy harvesting cognitive radio. $I E E E$ Commun. Lett., 1(5), 500-503.

47. Park, S., \& Hong, D. (2013). Optimal spectrum access for energy harvesting cognitive radio networks. IEEE Trans. Wireless Commun., 12(12), 6166-6179.

48. Varan, B., \& Yener, A. (2017). Online transmission policies for cognitive radio networks with energy harvesting secondary users. In Proc. IEEE Wireless Commun. and Networking Conf..

49. Kosta, A., Pappas, N., Ephremides, A., \& Angelakis, V. (2018). Age of information and throughput in a shared access network with heterogeneous traffic. arXiv preprint arXiv:1806.08776.

50. Leng, S., \& Yener, A. (2019). Minimizing age of information for an energy harvesting cognitive radio. In Proc. 
IEEE Wireless Commun. and Networking Conference.

51. Leng, S., \& Yener, A. (2019). Impact of Imperfect Spectrum Sensing on Age of Information in Energy Harvesting Cognitive Radios. IEEE International Conference on Communications (ICC).

52. Liu, B., Hua, C., \& Gu, P. (2019). Age of Information Aware Channel Allocation for Wireless Industrial Networks. International Conference on Wireless Communications and Signal Processing.

53. Zheng, K., Liu, X., Liu, X., \& Zhu Y. (2019). Hybrid Overlay-Underlay Cognitive Radio Networks With Energy Harvesting. IEEE Trans. Commun., 67, 4669-4682.

54. Karaca, H. M. (2020). Throughput Maximization of Multichannel Allocation Mechanism under Interference Constraint $\mathrm{f}$ or Hybrid Overlay/underlay Cognitive Radio Networks with Energy Harvesting. Wireless Networks ,26, 3905-3928

55. Usman, M., \& Koo, I. (2014). Access strategy for hybrid underlay-overlay cognitive radios with energy harvesting. IEEE Sensors Journal, 14(9), 3164-3173.

56. Miao, M., \& Danny, T. (2010). Cross-layer throughput optimization in cognitive radio networks with SINR constraints. International Journal of Digital Multimedia Broadcasting, https://doi.org/10.1155/2010/985458.

57. Sac, H., Bacinoglu, T., Uysal-Biyikoglu, E., \& Durisi, G. (2018). Age-optimal channel coding blocklength for an M/G/1 queue with HARQ. Proc. 19th Int. Workshop Signal Process. Adv. Wireless Commun.(SPAWC), 486-490.

Figures

Figure 1 fig_1.eps Average Age of Information against different SINR (threshold) values for Poisson distribution density of SUs of 0.0003 on the area of $250 \times 250 \mathrm{~m}^{2}$ around the PU and average percentage of SUs in harvesting zone of about $2.9 \%$.

Figure 2 fig_2.eps Channel Distribution Performance within increasing time slots against different SINR (threshold) values.

Figure 3 fig_3.eps Average Age of Information of Proposed and Greedy Algorithms against different SINR (threshold) values.

Figure 4 fig_4.eps Channel Distribution Performance of Proposed and Greedy Algorithms against different SINR (threshold) values.

Figure 5 fig_5.eps ChDistFairness against different SINR (threshold) values for Poisson distribution density of STs of 0.0003 in the $250 \times 250 \mathrm{~m}^{2}$ and 0.001 in $150 \times 150 \mathrm{~m}^{2}$ area around the PT. Average percentage of STs in harvesting zone are about $3 \%$ and $12 \%$ respectively.

Figure 6 fig_6.eps Average Aol values for Poisson distribution density of STs of 0.0003 in the 250 $\times 250 \mathrm{~m}^{2}$ and 0.001 in $150 \times 150 \mathrm{~m}^{2}$ area around the PT.

Figure 7 fig_8.eps ChDistFairness against different SINR (threshold) values for STs/PTs ratio of $30 / 8$ and $17 / 4$ at the same percentage of harvesting STs.

\author{
Abbreviations \\ Aol Age of Information \\ AoS Age of Synchronization
}


Figure 8 fig_7.eps Average Aol values against different SINR (threshold) values for STs/PTs ratio of $30 / 8$ and $17 / 4$ at the same percentage of harvesting STs.

Figure 9 fig_9.eps Average Aol values against different SINR (threshold) values for Poisson distribution density of STs of 0.0008 in a $100 \times 100 \mathrm{~m}^{2}$ area around the PT and average percentage of STs in the harvesting zone of about $3 \%$.

Figure 10 fig_10.eps ChDistFairness against different SINR (threshold) values for Poisson distribution density of STs of 0.0008 in a $100 \times 100 \mathrm{~m}^{2}$ area around the PT and average percentage of STs in the harvesting zone of about $3 \%$.

Figure 11 fig_11.eps Average Aol values against different SINR (threshold) values for Poisson distribution density of STs of 0.0008 in a $100 \times 100 \mathrm{~m}^{2}$ area around the PT and average percentage of STs in the harvesting zone of about $3 \%$.

Figure 12 fig_12.eps Channel Distribution Performance within increasing time slots.

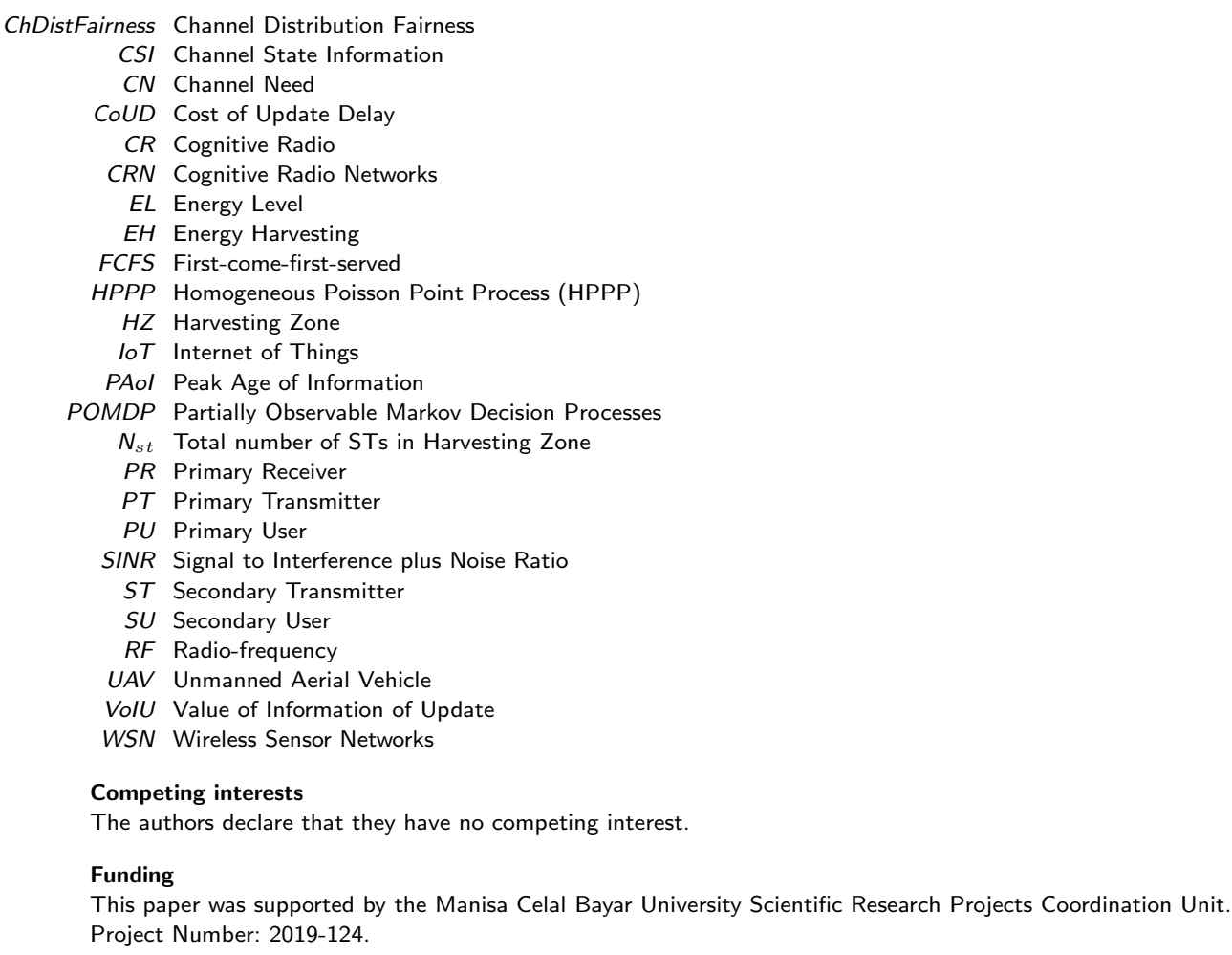

Acknowledgements

As the author of the article, I would like to thank the anonymous reviewers for their insightful comments.

1 Availibility of data and materials

The authors confirm that the data supporting the findings of this study will be supplied upon request. 


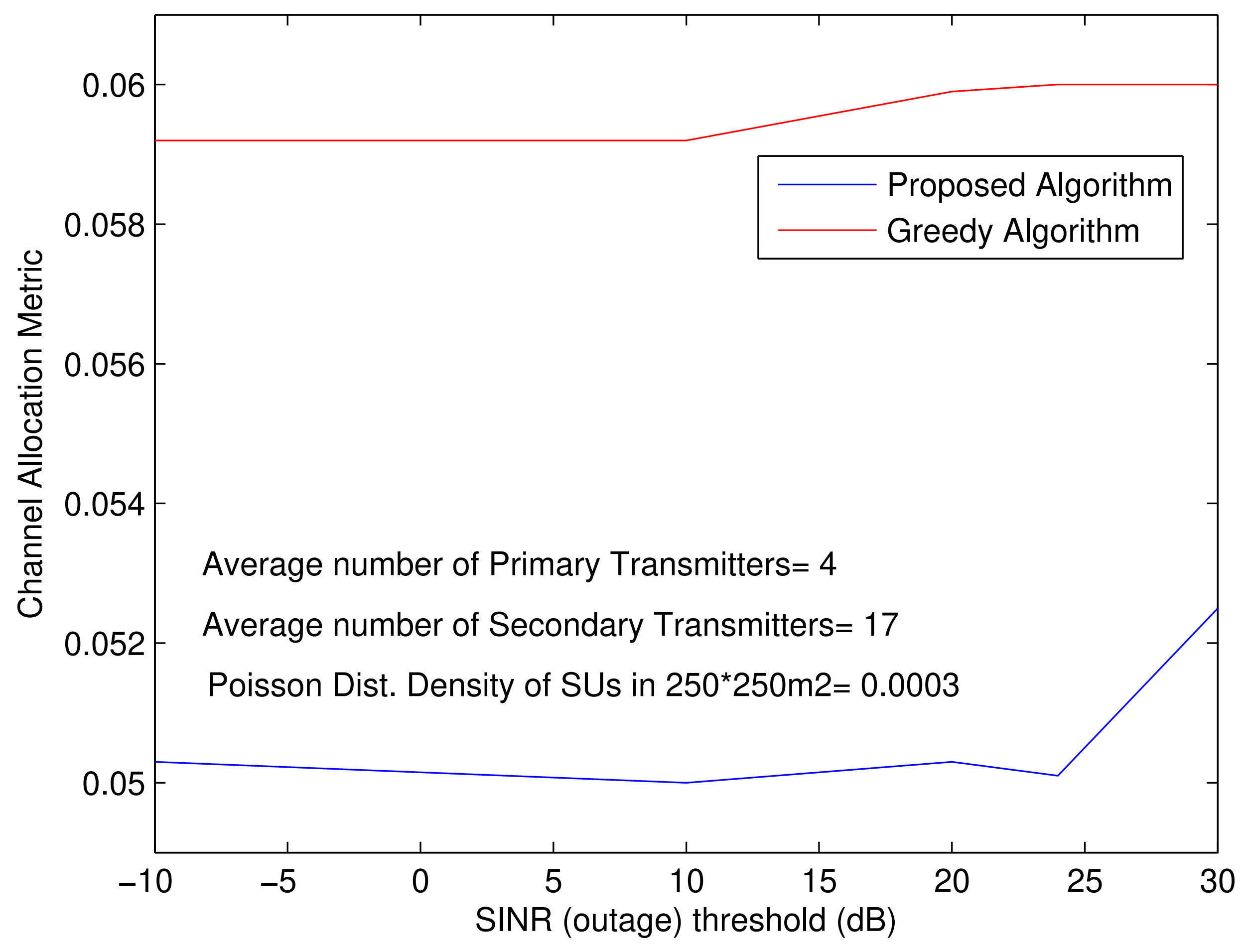




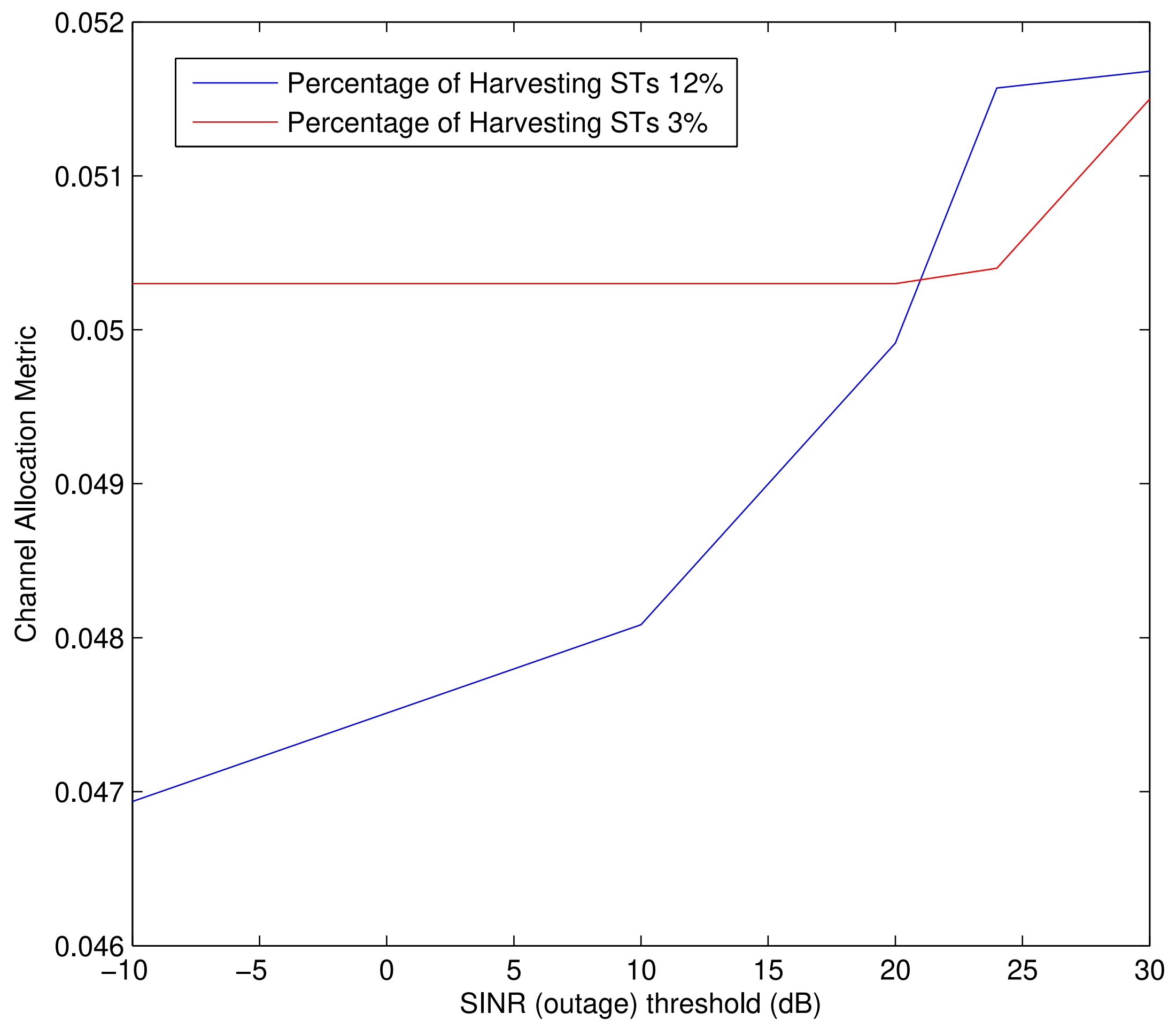




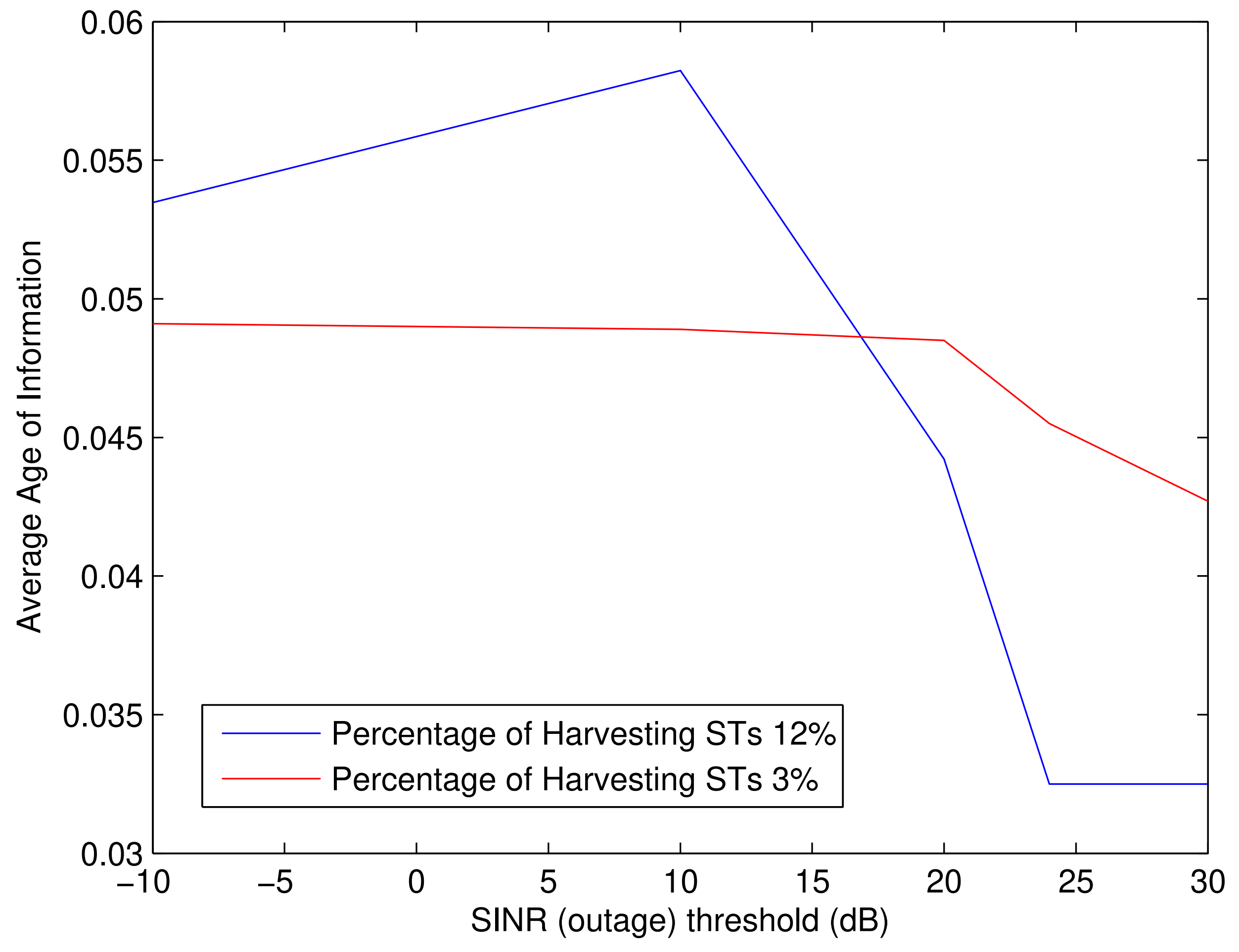




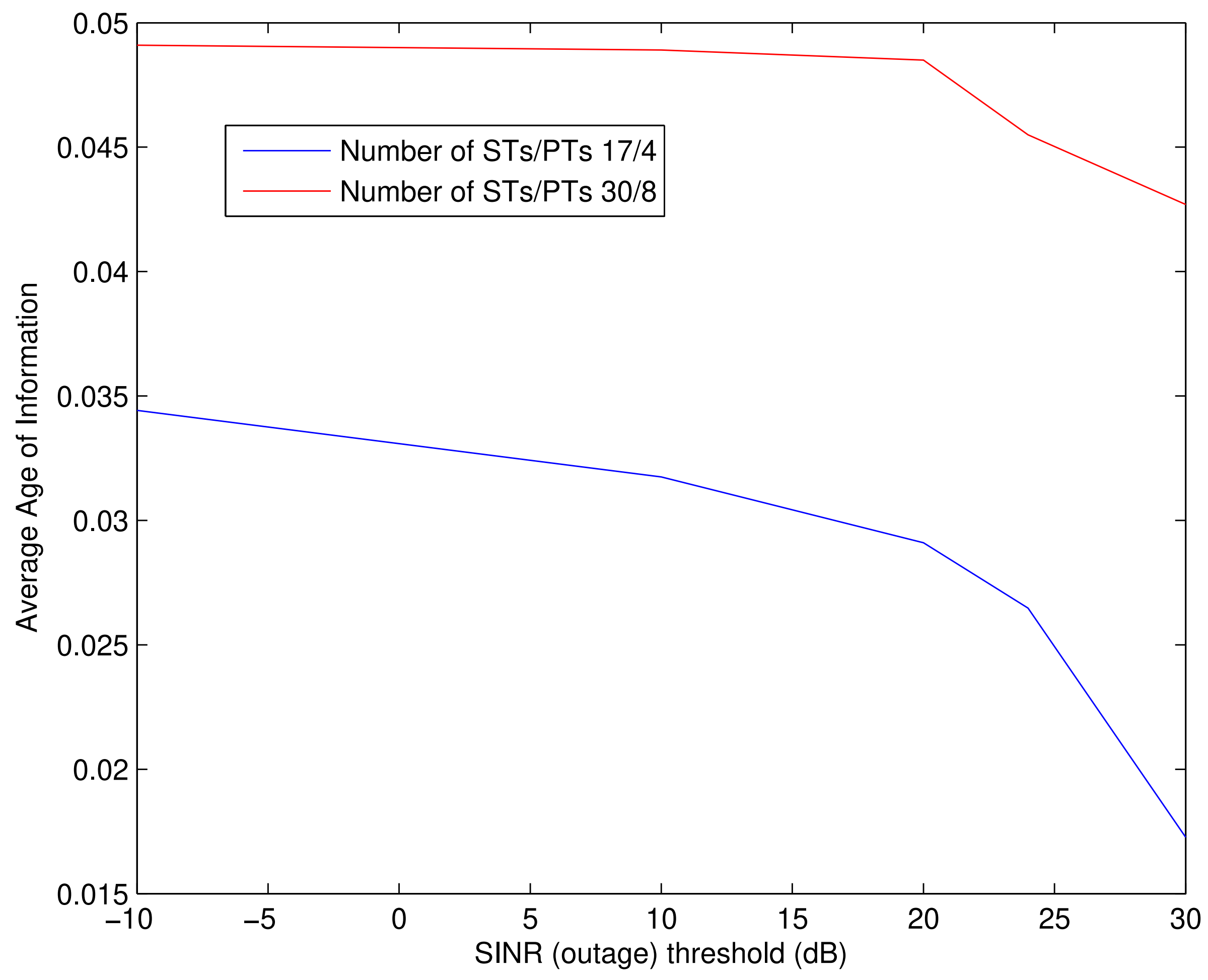




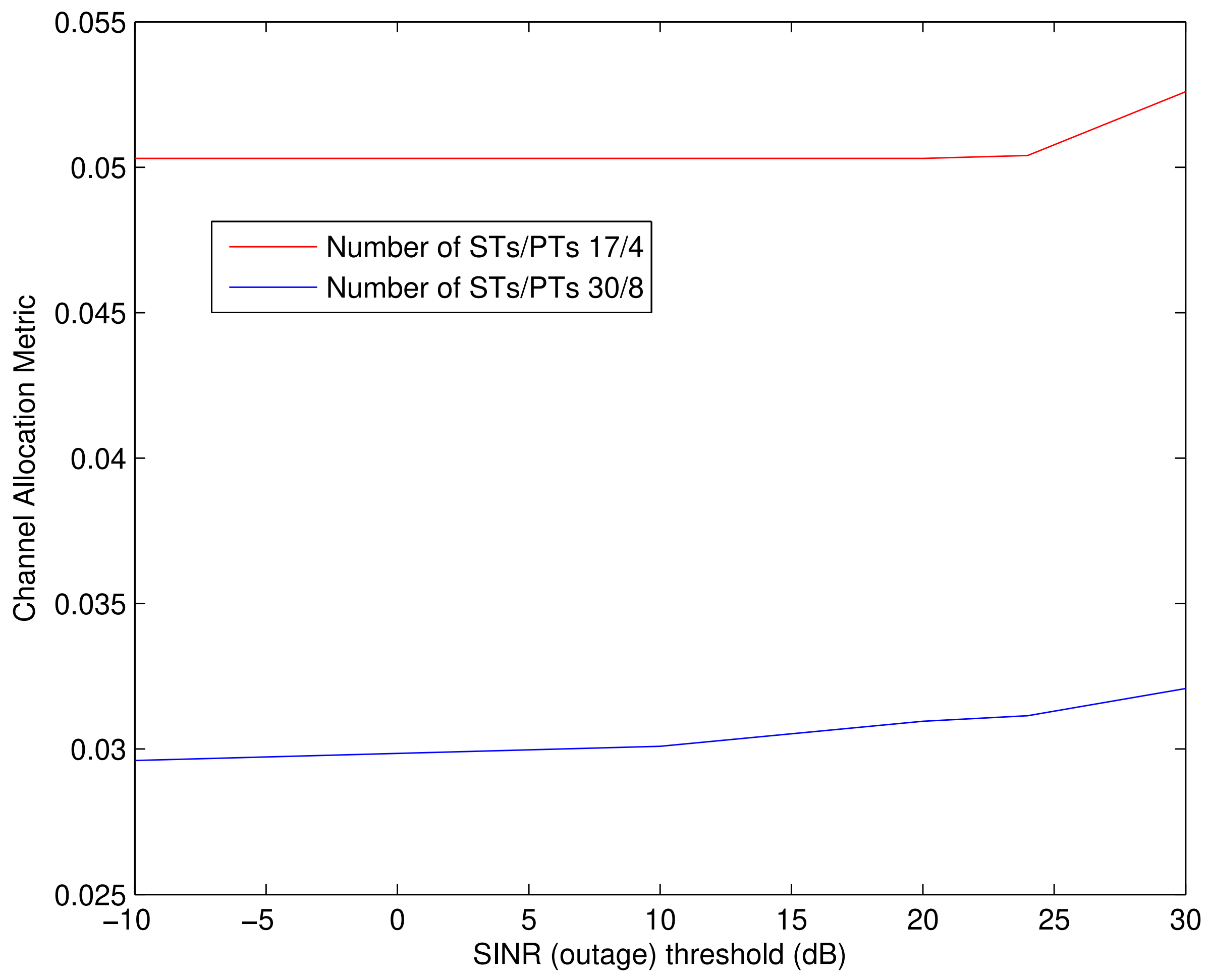




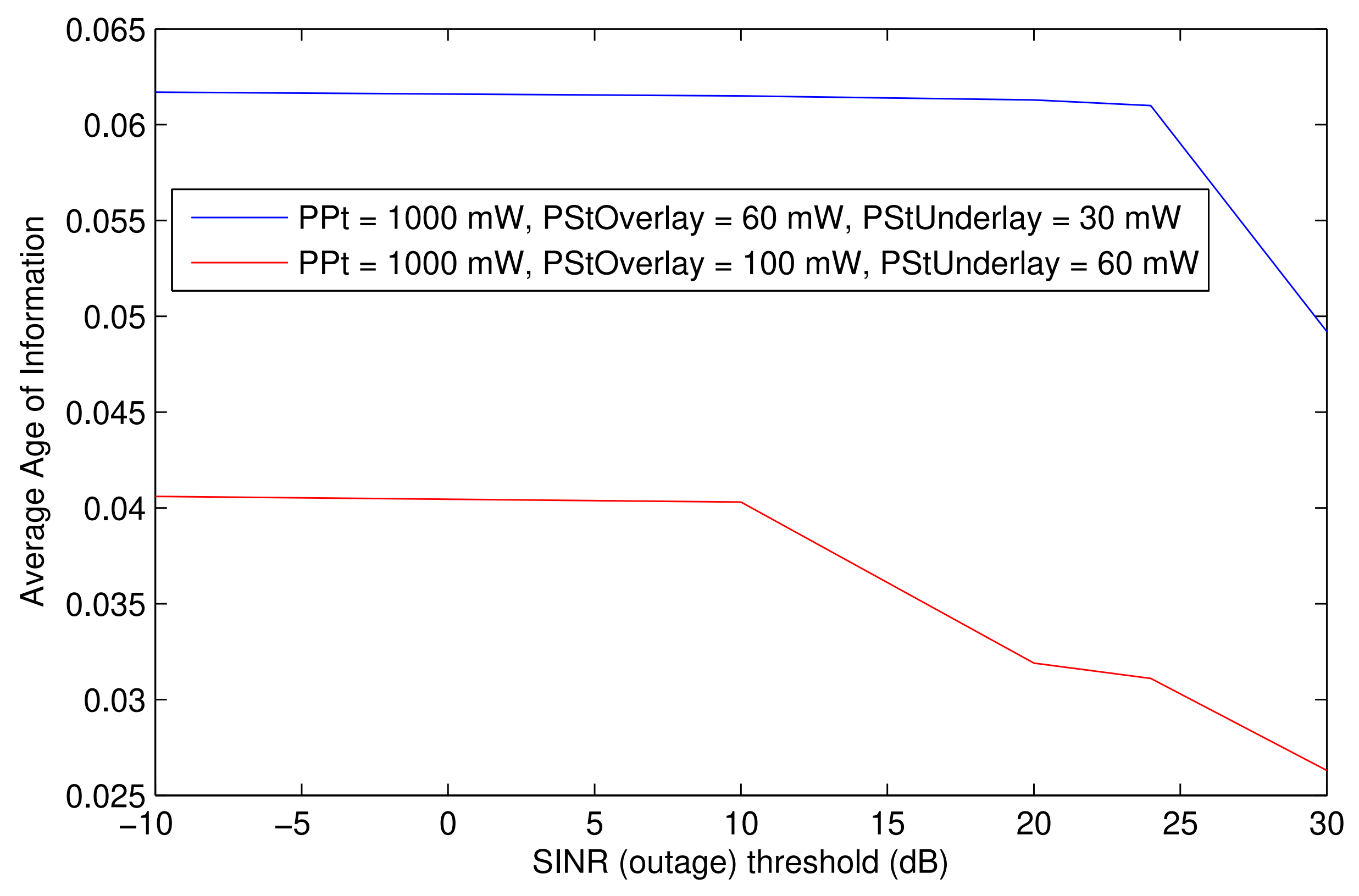




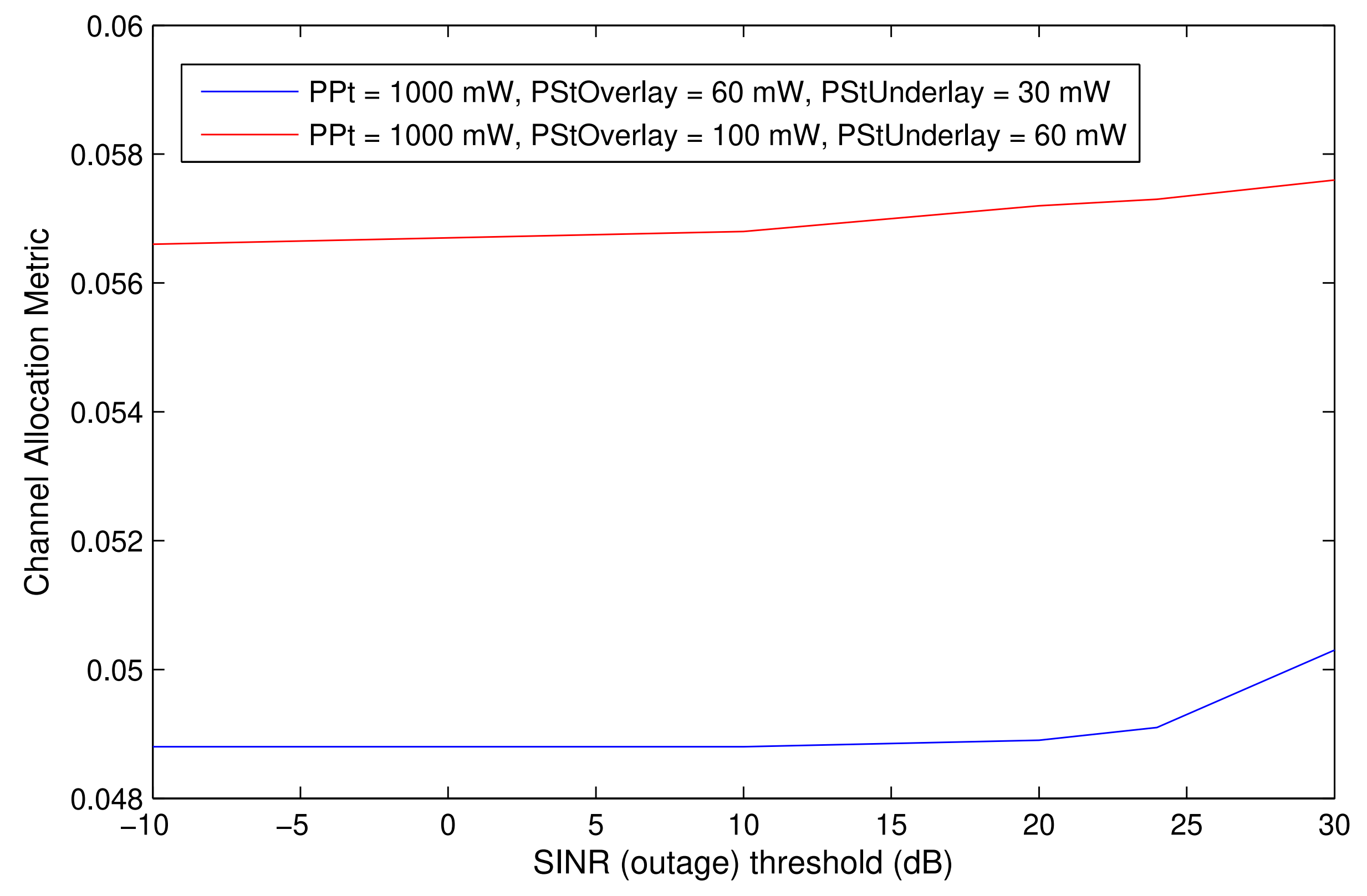




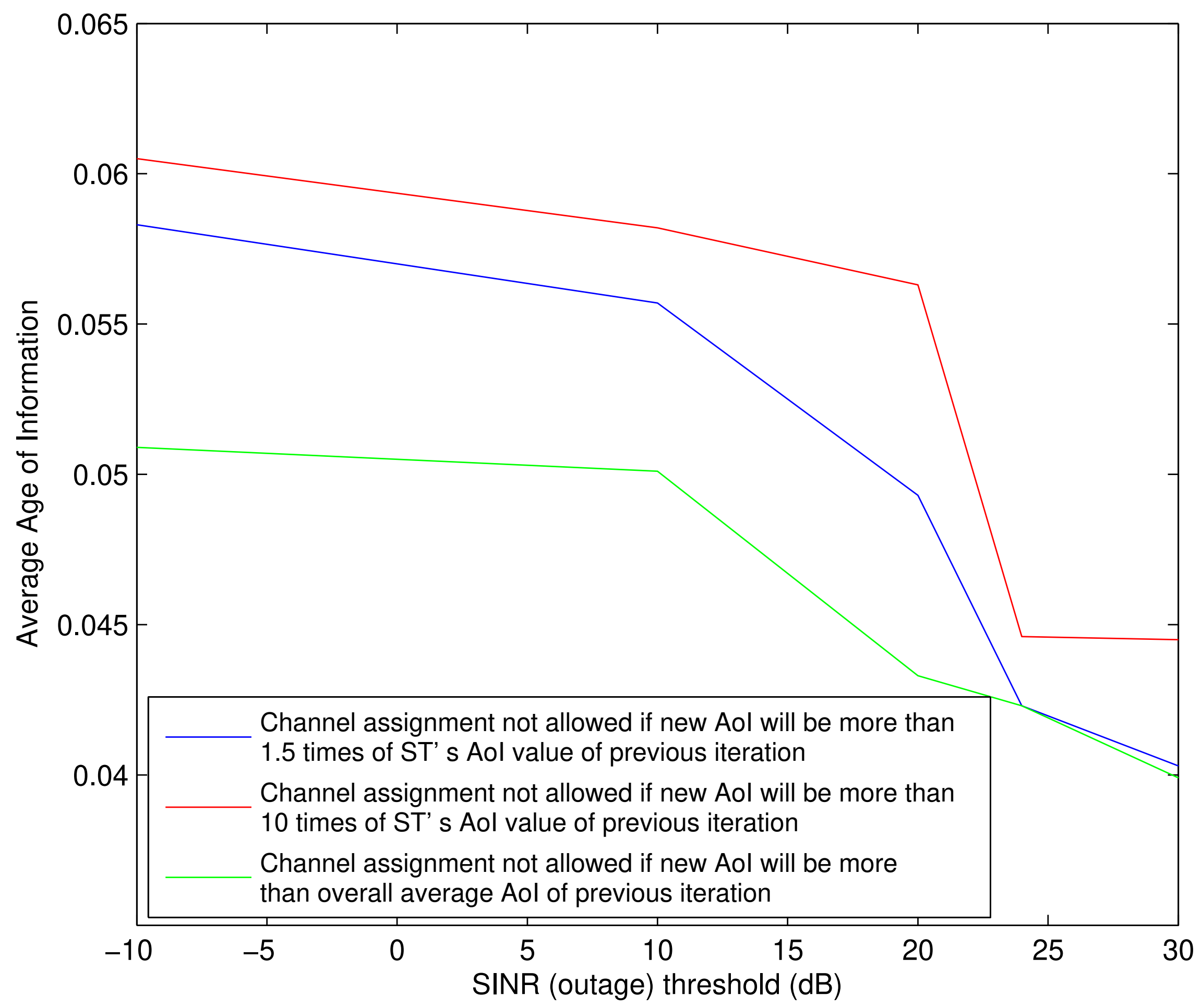




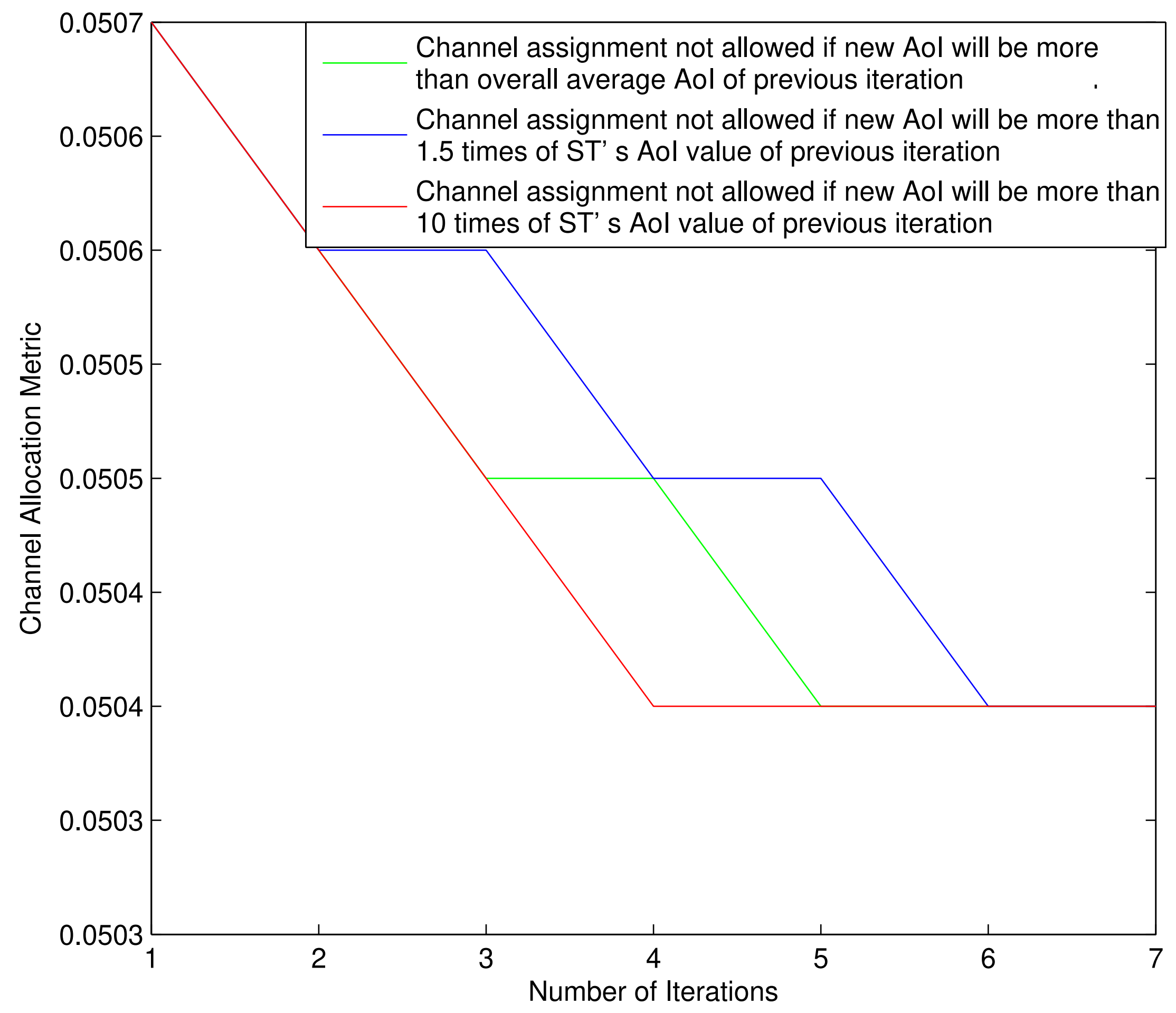




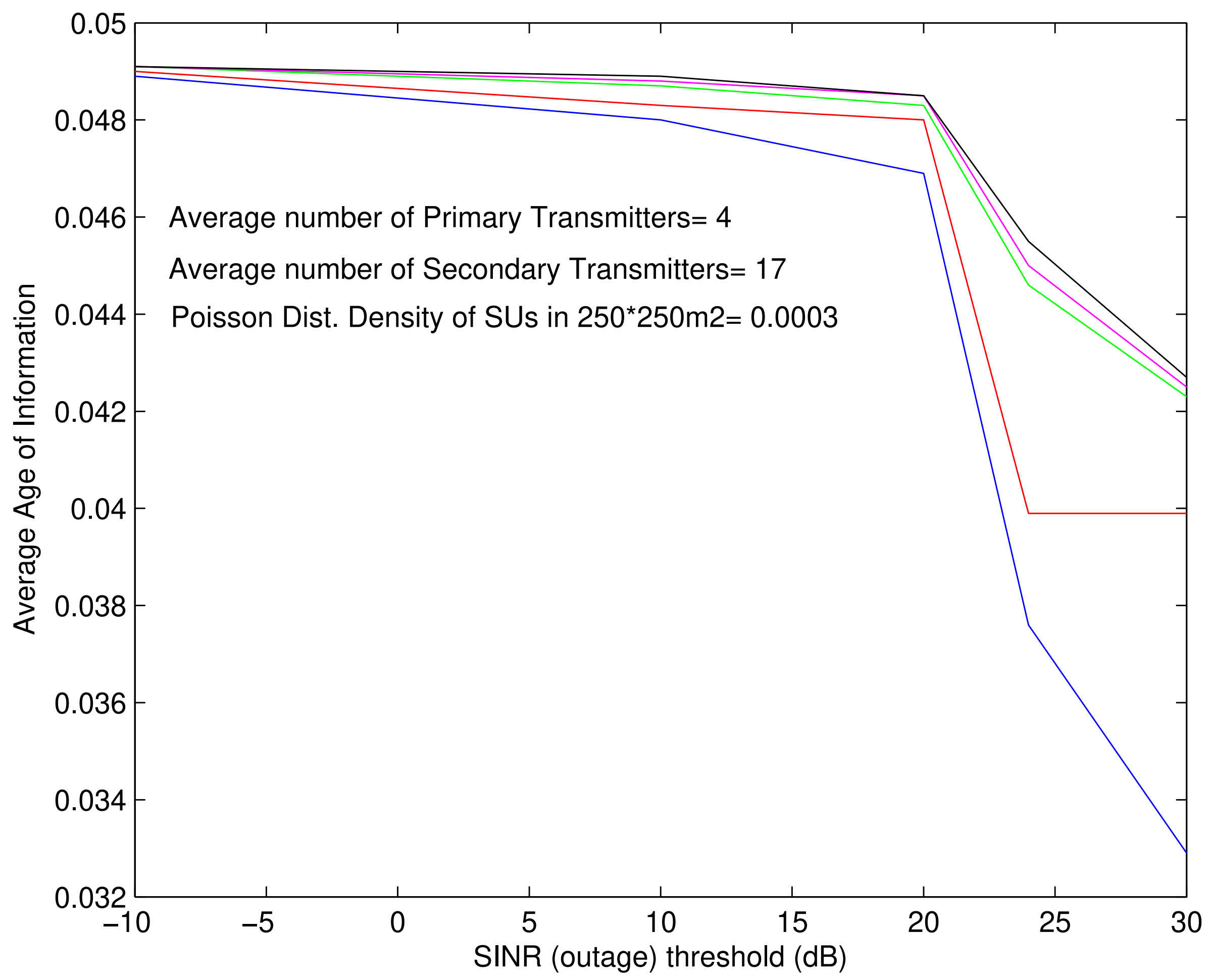




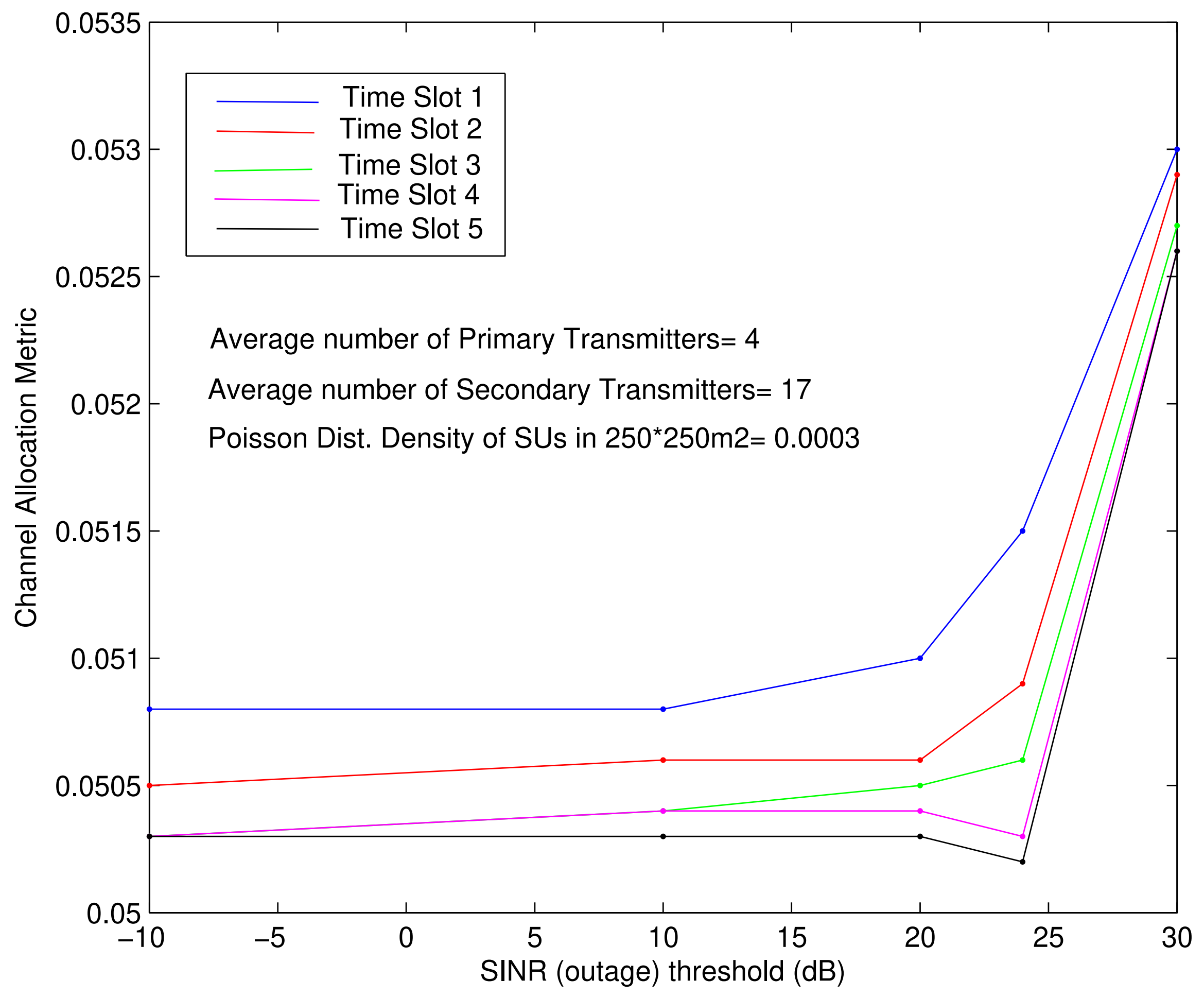




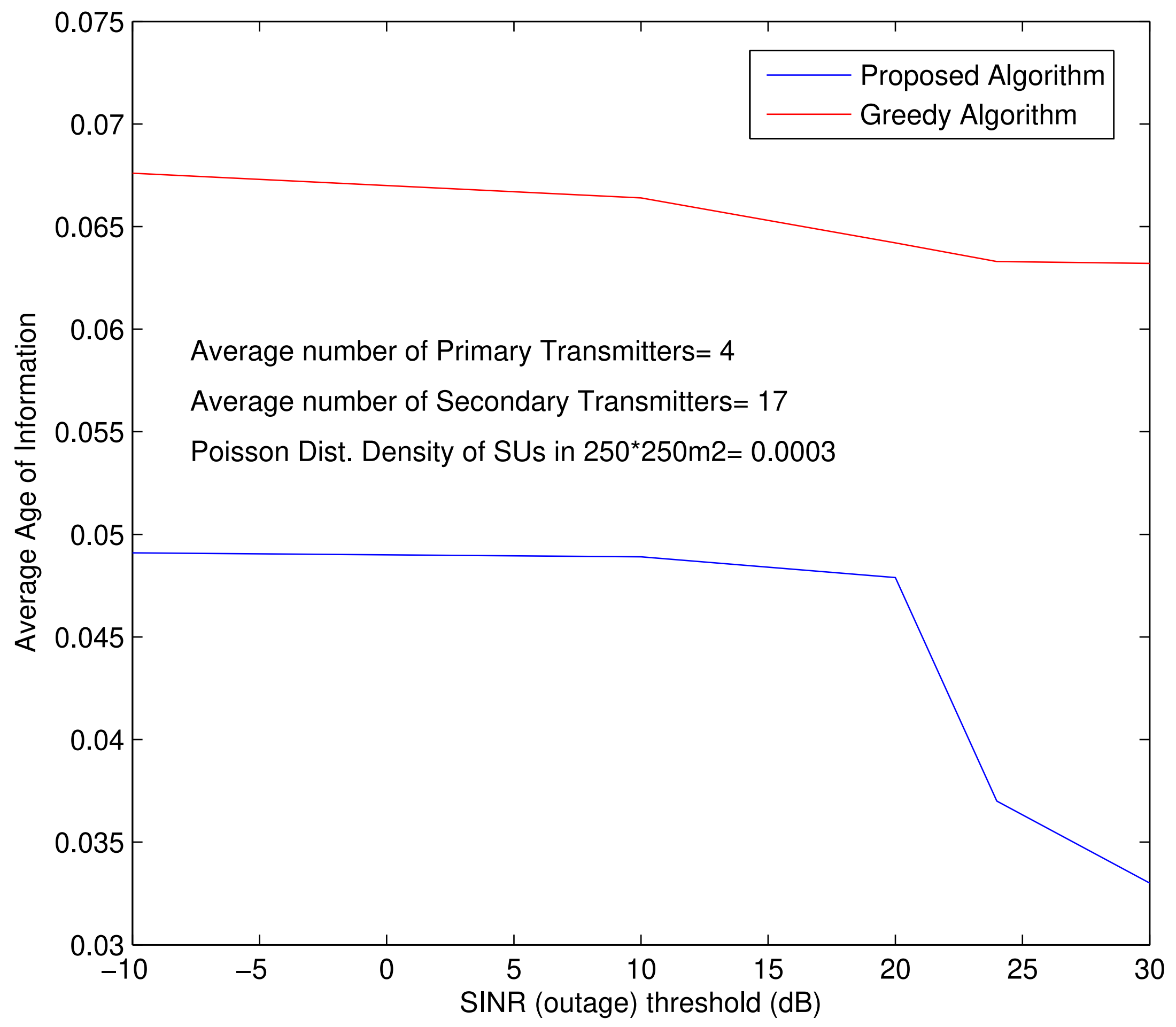


Figures

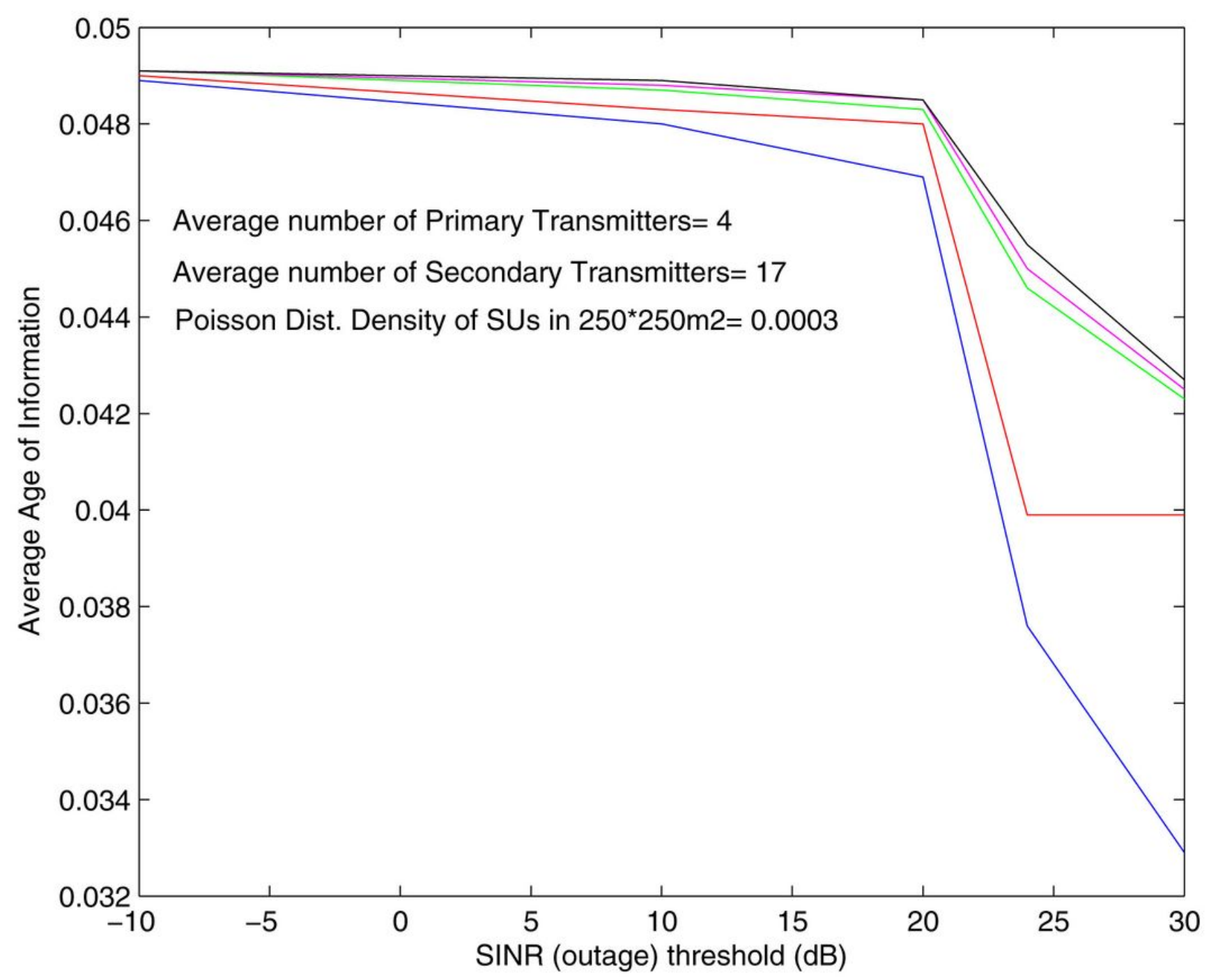

Figure 1

Average Age of Information against different SINR (threshold) values for Poisson distribution density of SUs of 0.0003 on the area of $250 \times 250 \mathrm{~m} 2$ around the PU and average percentage of SUs in harvesting zone of about $2.9 \%$. 


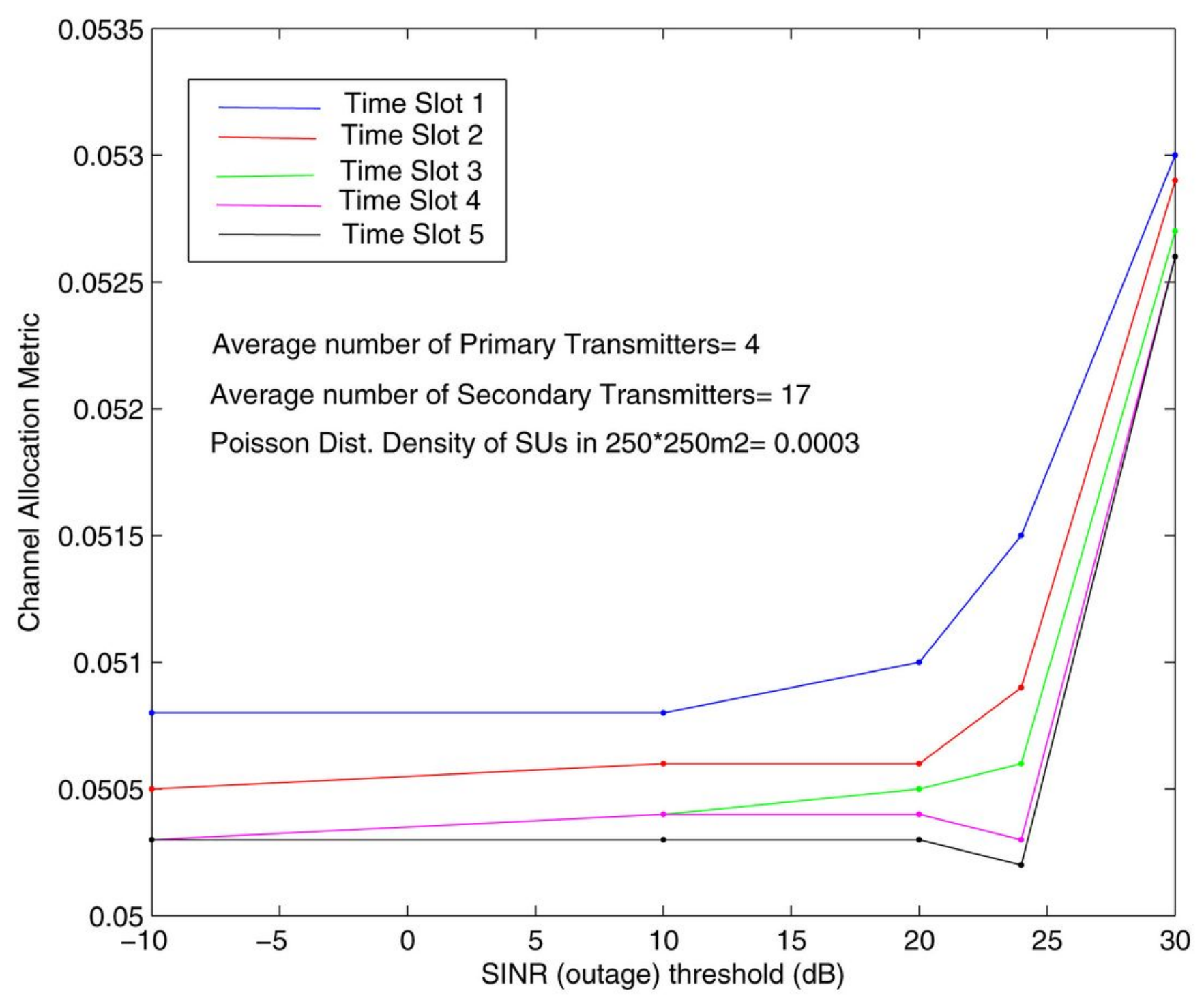

Figure 2

Channel Distribution Performance within increasing time slots against different SINR (threshold) values. 


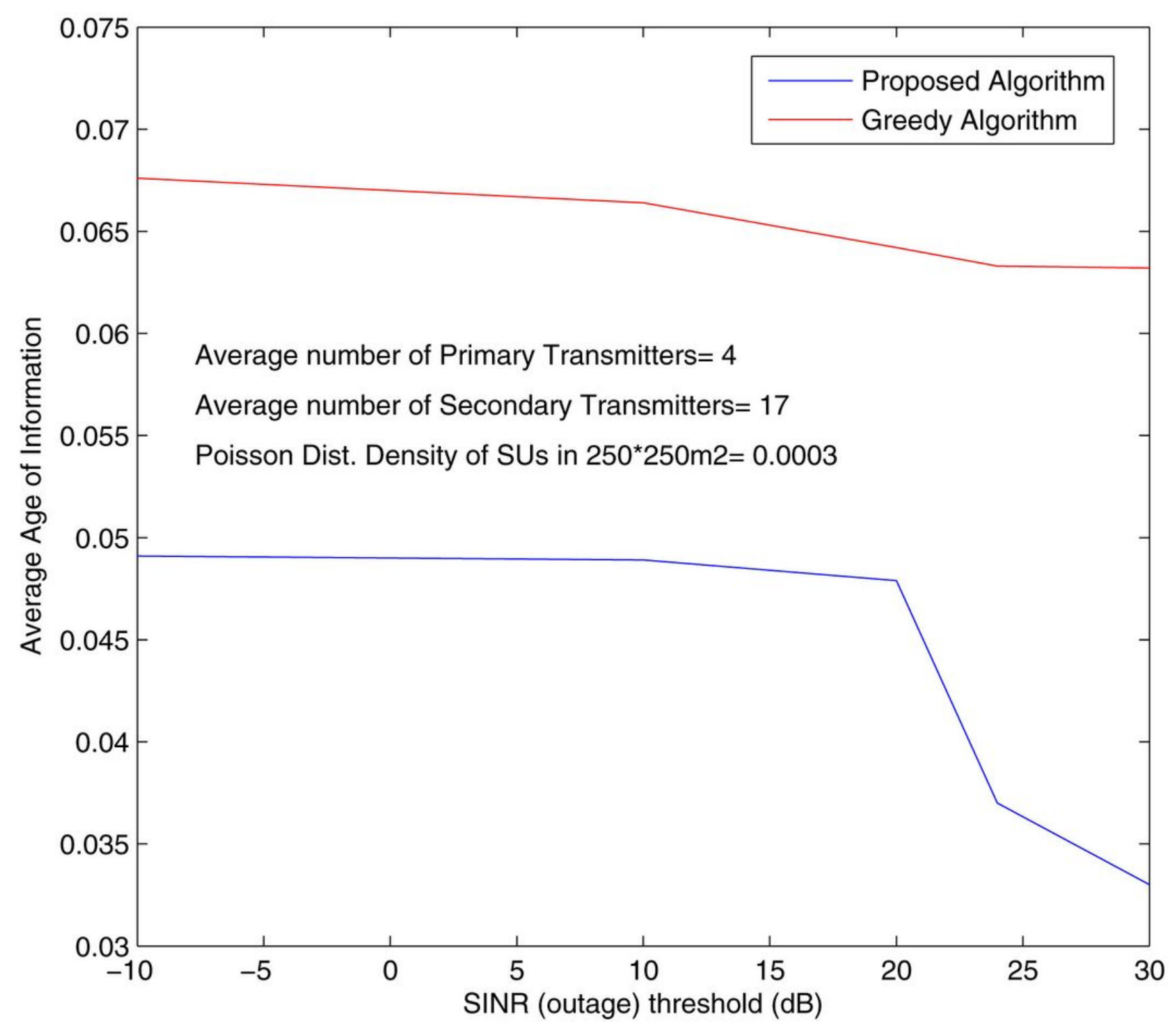

Figure 3

Average Age of Information of Proposed and Greedy Algorithms against different SINR (threshold) values. 


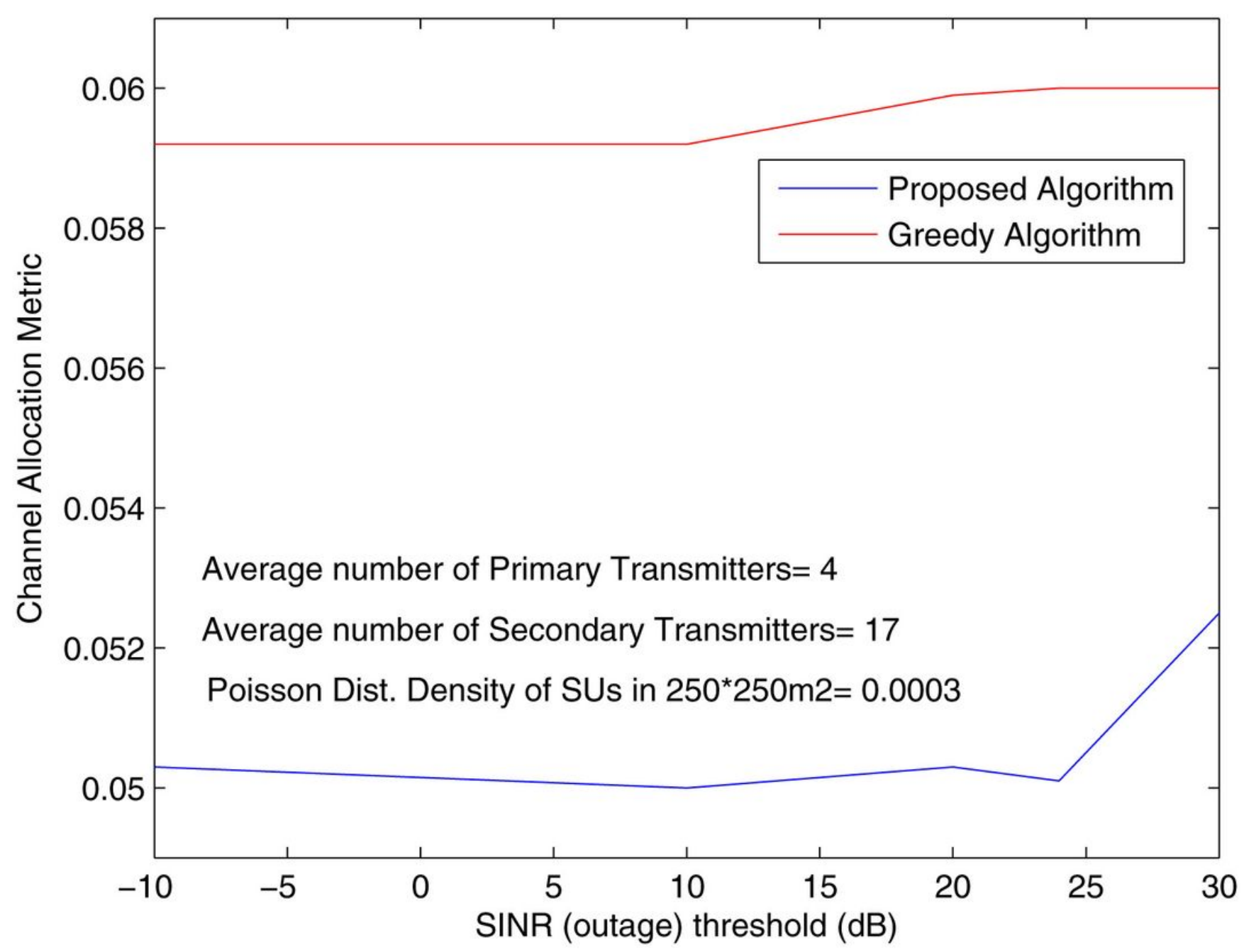

Figure 4

Channel Distribution Performance of Proposed and Greedy Algorithms against different SINR (threshold) values. 


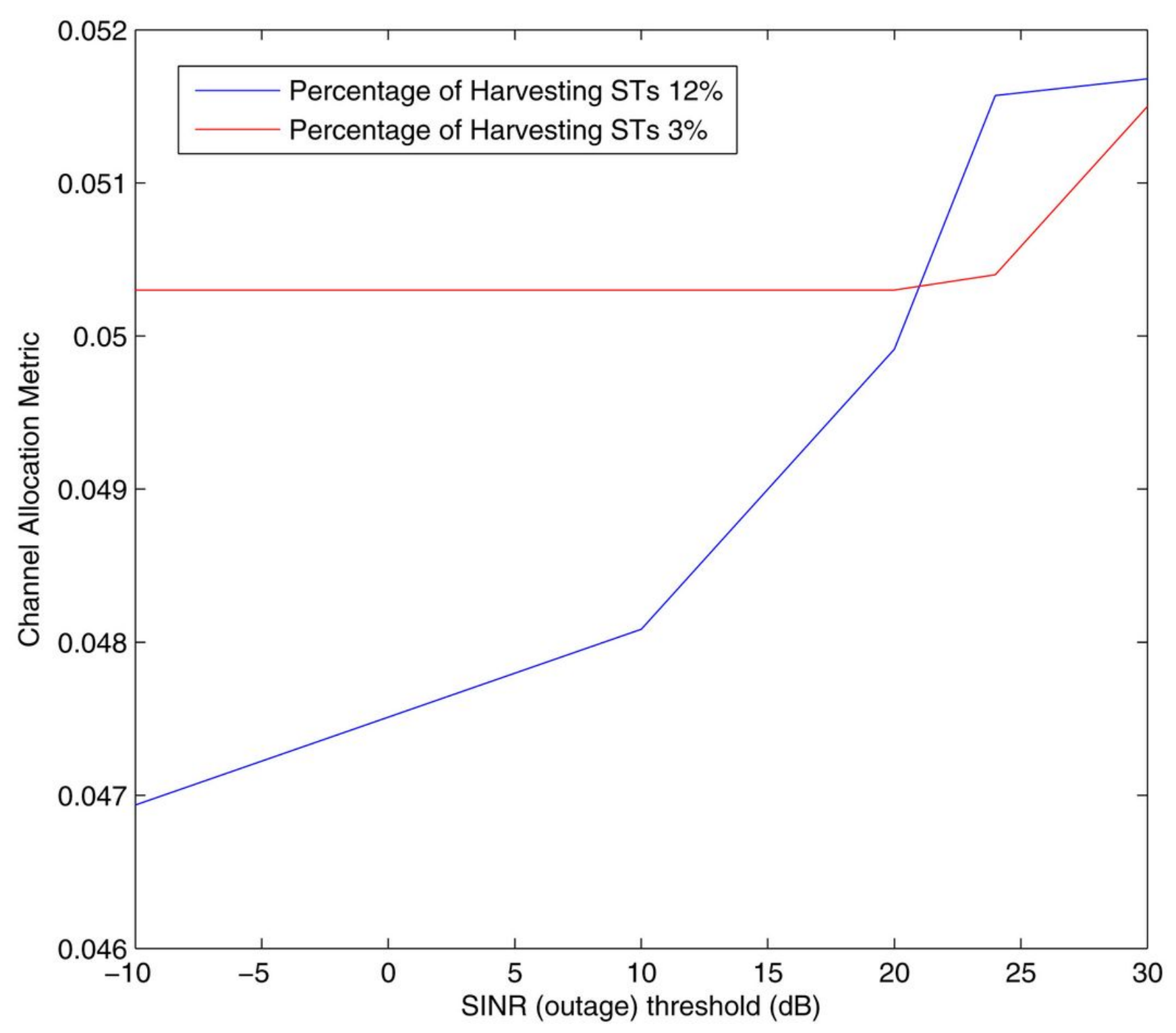

Figure 5

ChDistFairness against different SINR (threshold) values for Poisson distribution density of STs of 0.0003 in the $250 \times 250 \mathrm{~m} 2$ and 0.001 in $150 \times 150 \mathrm{~m} 2$ area around the PT. Average percentage of STs in harvesting zone are about $3 \%$ and $12 \%$ respectively. 


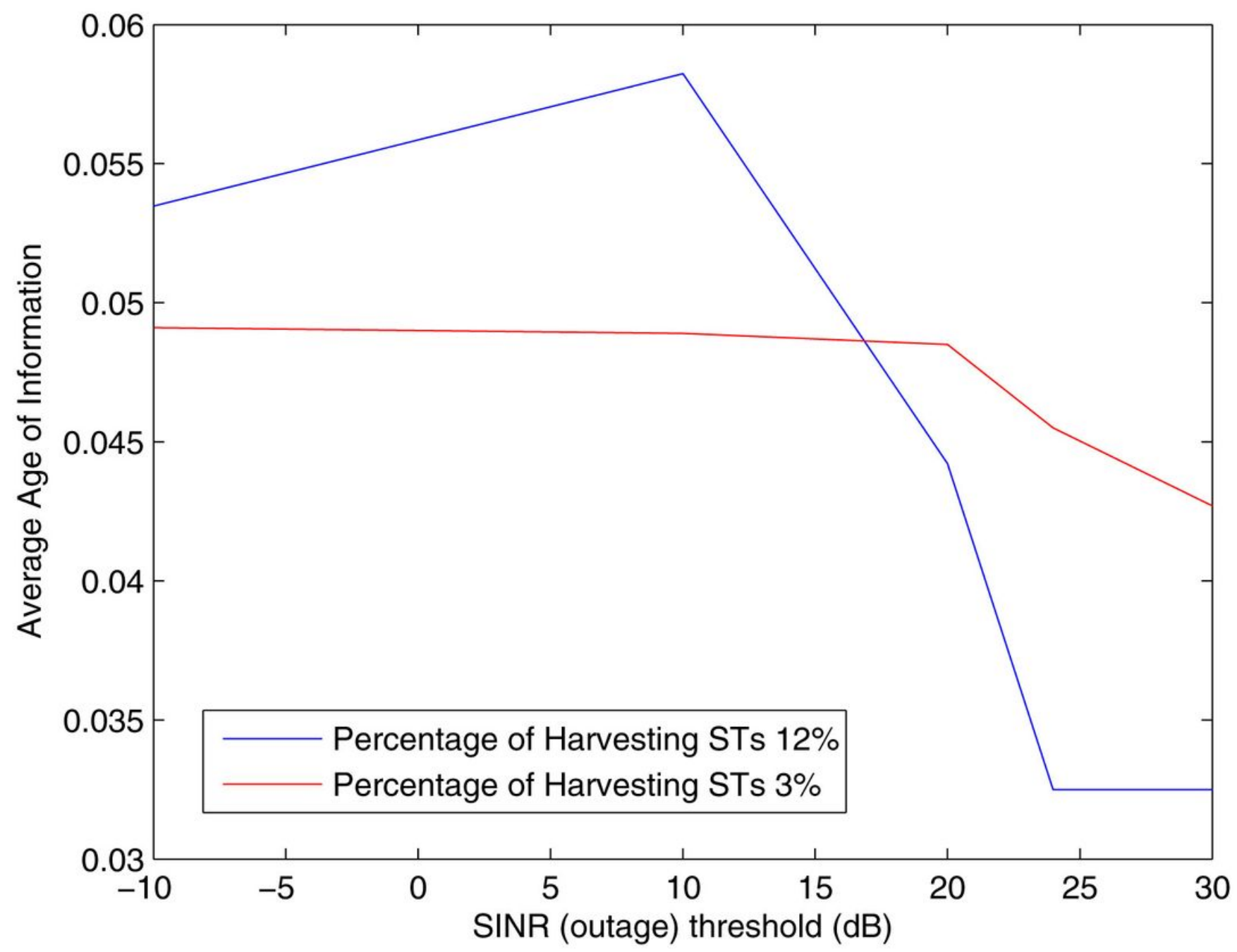

Figure 6

Average Aol values for Poisson distribution density of STs of 0.0003 in the $250 \times 250 \mathrm{~m} 2$ and 0.001 in $150 \times 150 \mathrm{~m} 2$ area around the PT. 


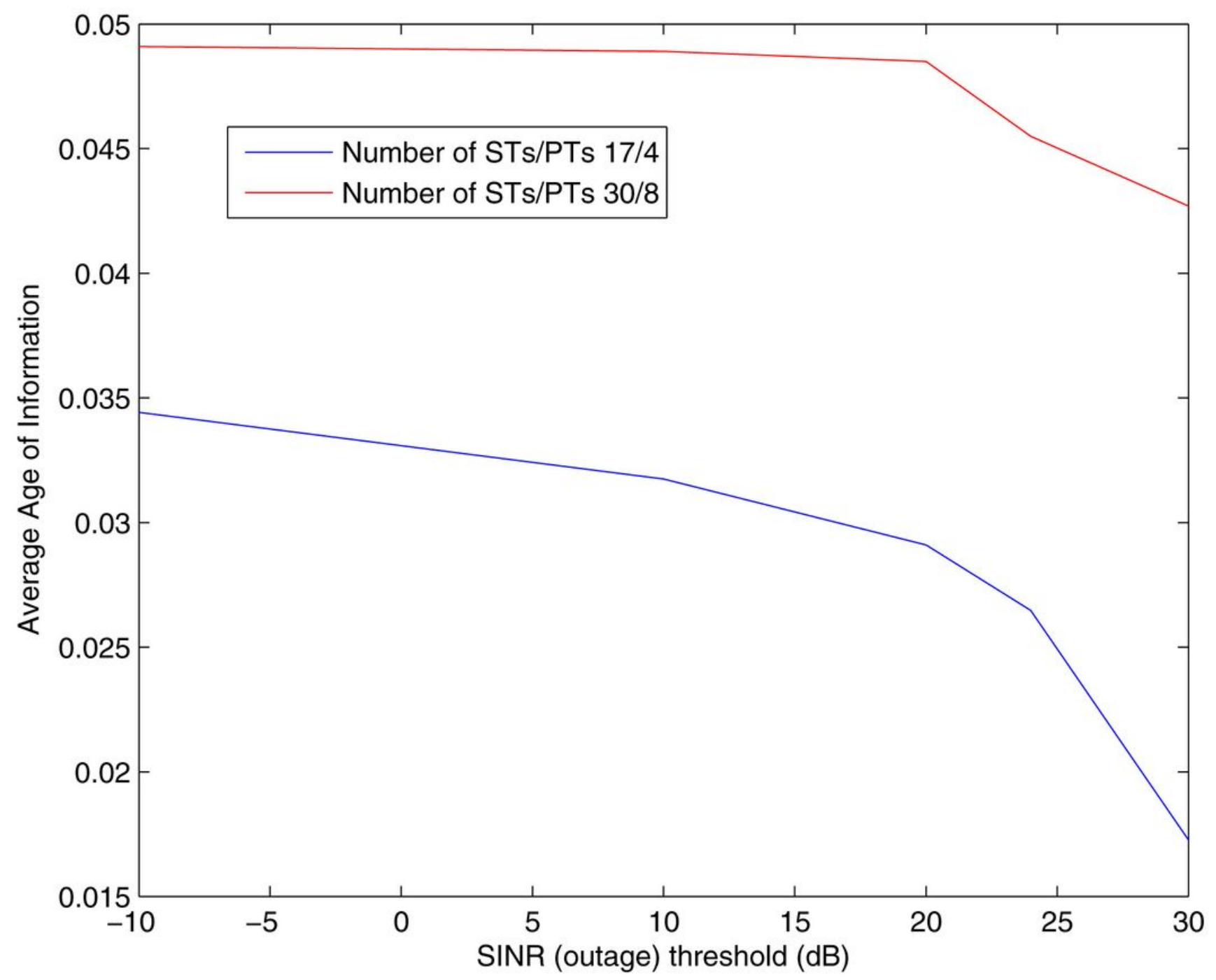

Figure 7

ChDistFairness against different SINR (threshold) values for STs/PTs ratio of 30/8 and 17/4 at the same percentage of harvesting STs. 


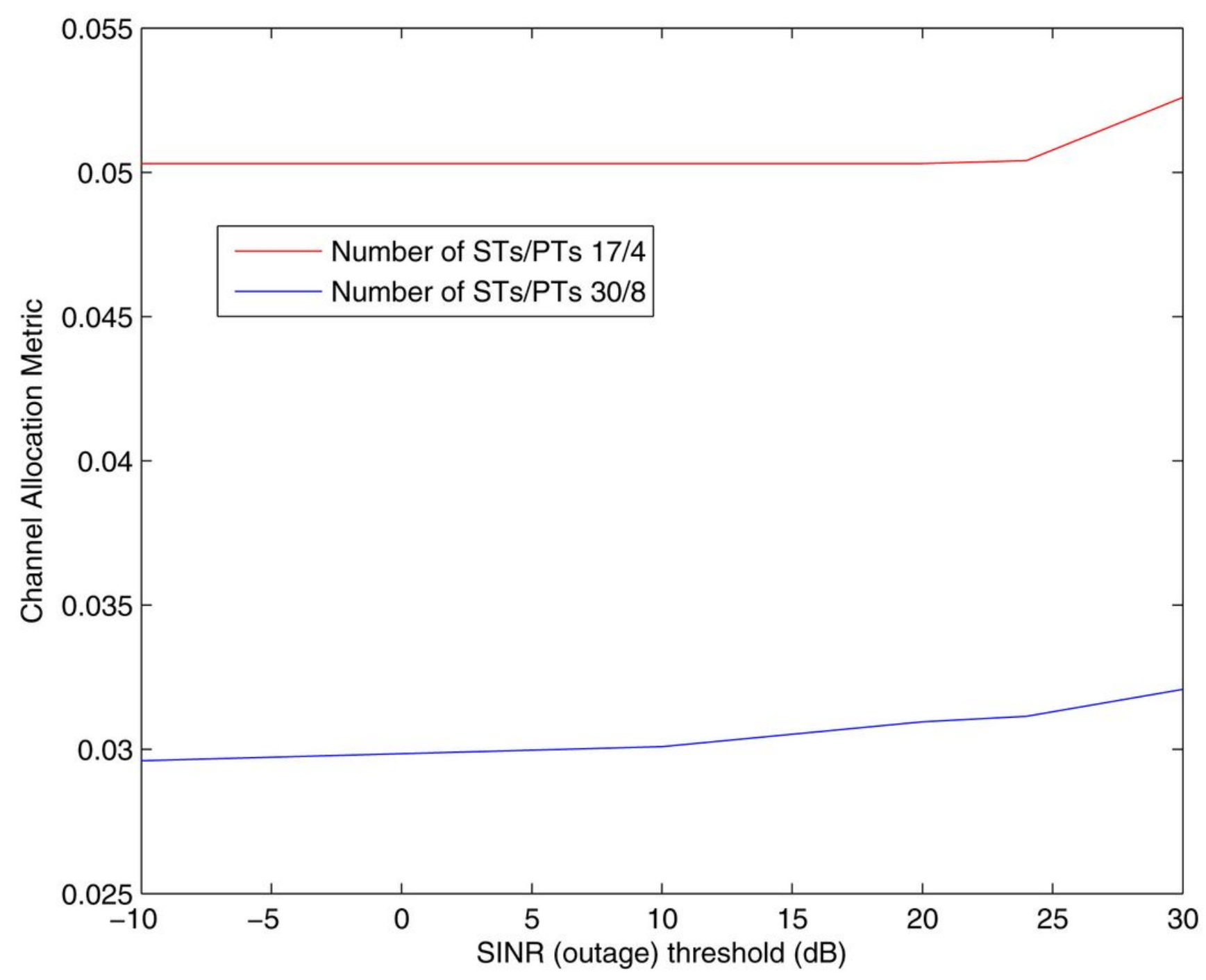

Figure 8

Average Aol values against different SINR (threshold) values for STs/PTs ratio of 30/8 and 17/4 at the same percentage of harvesting STs. 


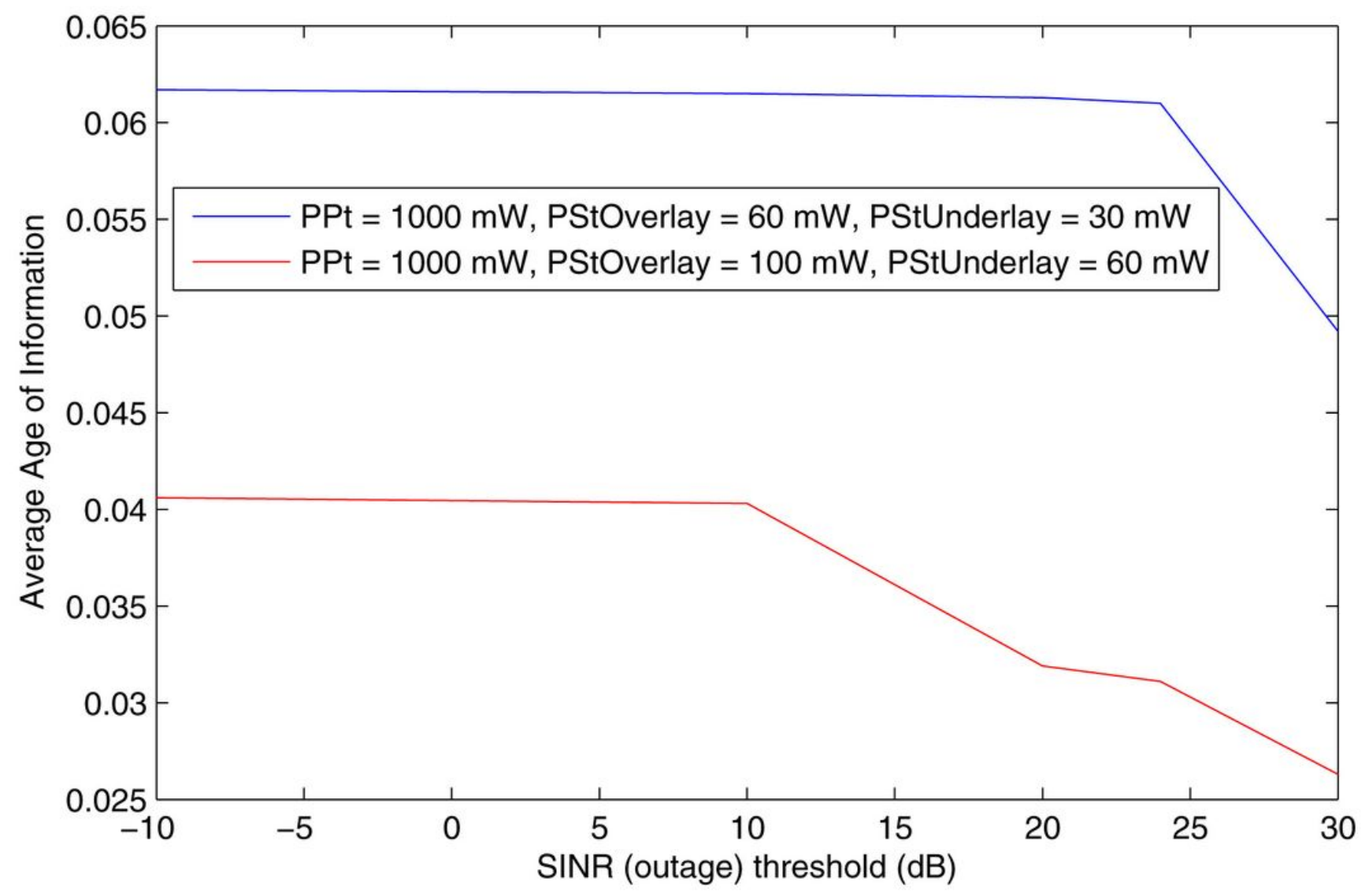

Figure 9

Average Aol values against different SINR (threshold) values for Poisson distribution density of STs of 0.0008 in a $100 \times 100 \mathrm{~m} 2$ area around the PT and average percentage of STs in the harvesting zone of about $3 \%$. 


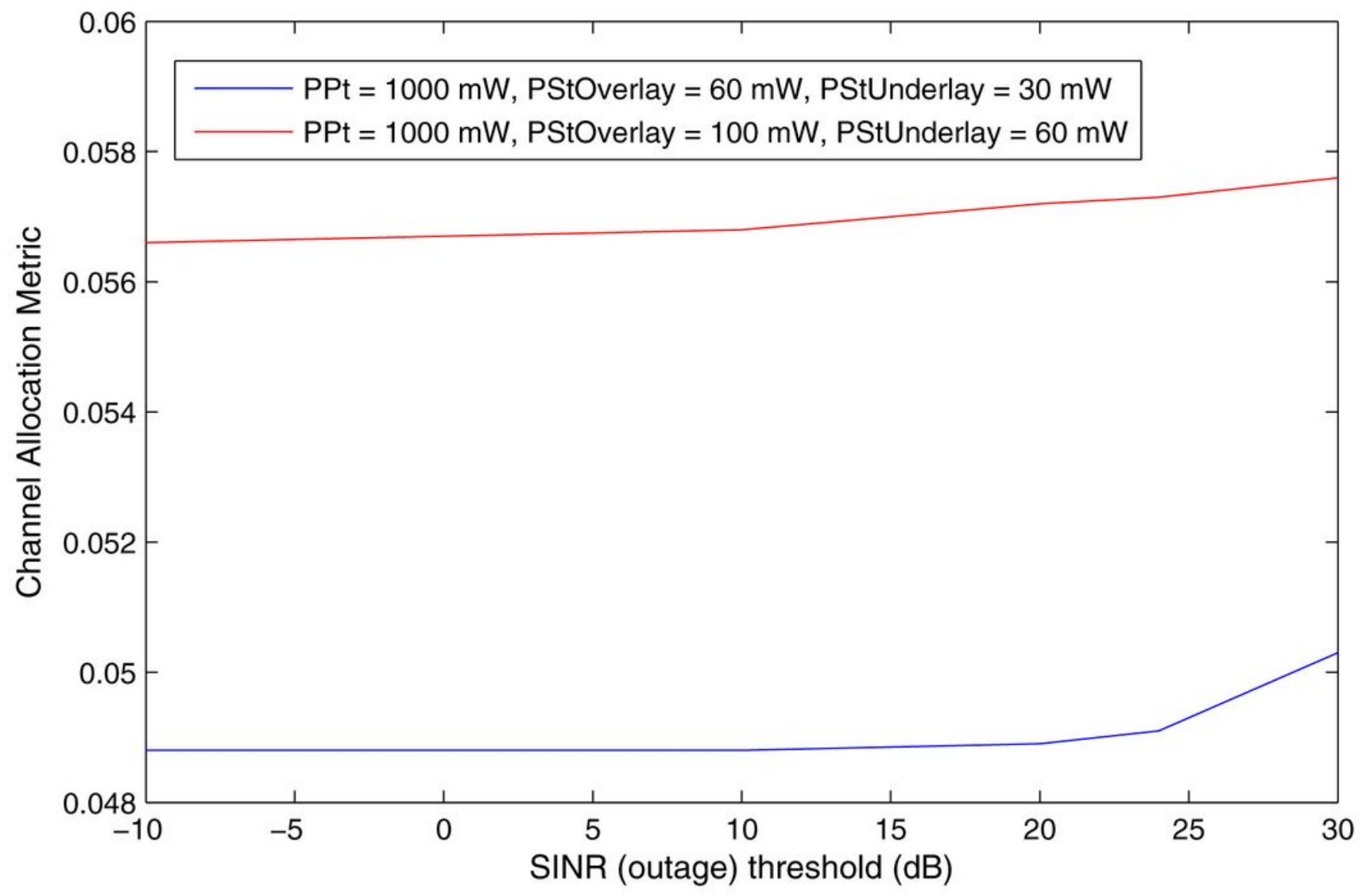

Figure 10

ChDistFairness against different SINR (threshold) values for Poisson distribution density of STs of 0.0008 in a $100 \times 100 \mathrm{~m} 2$ area around the PT and average percentage of STs in the harvesting zone of about $3 \%$. 


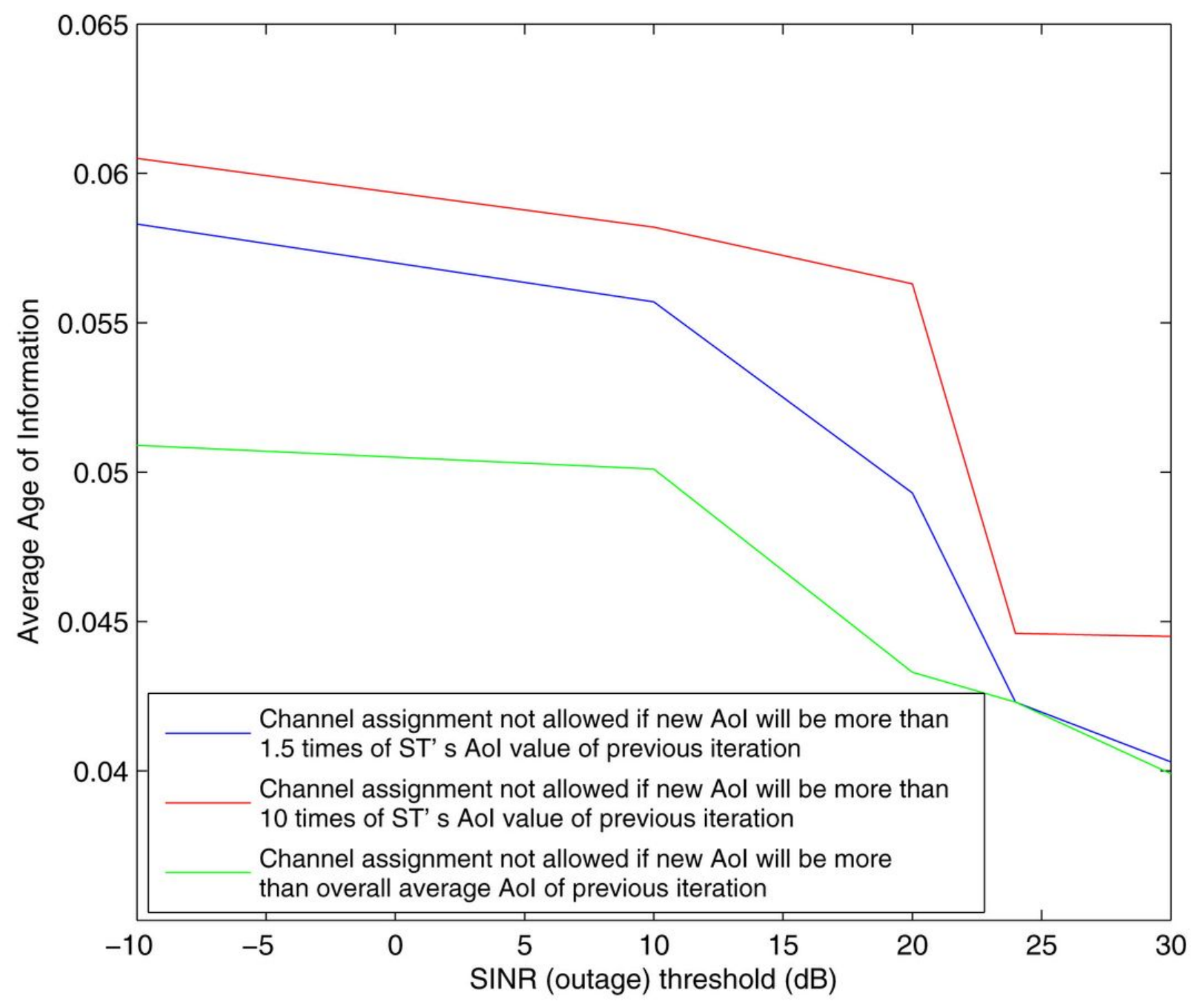

Figure 11

Average Aol values against different SINR (threshold) values for Poisson distribution density of STs of 0.0008 in a $100 \times 100 \mathrm{~m} 2$ area around the PT and average percentage of STs in the harvesting zone of about $3 \%$. 


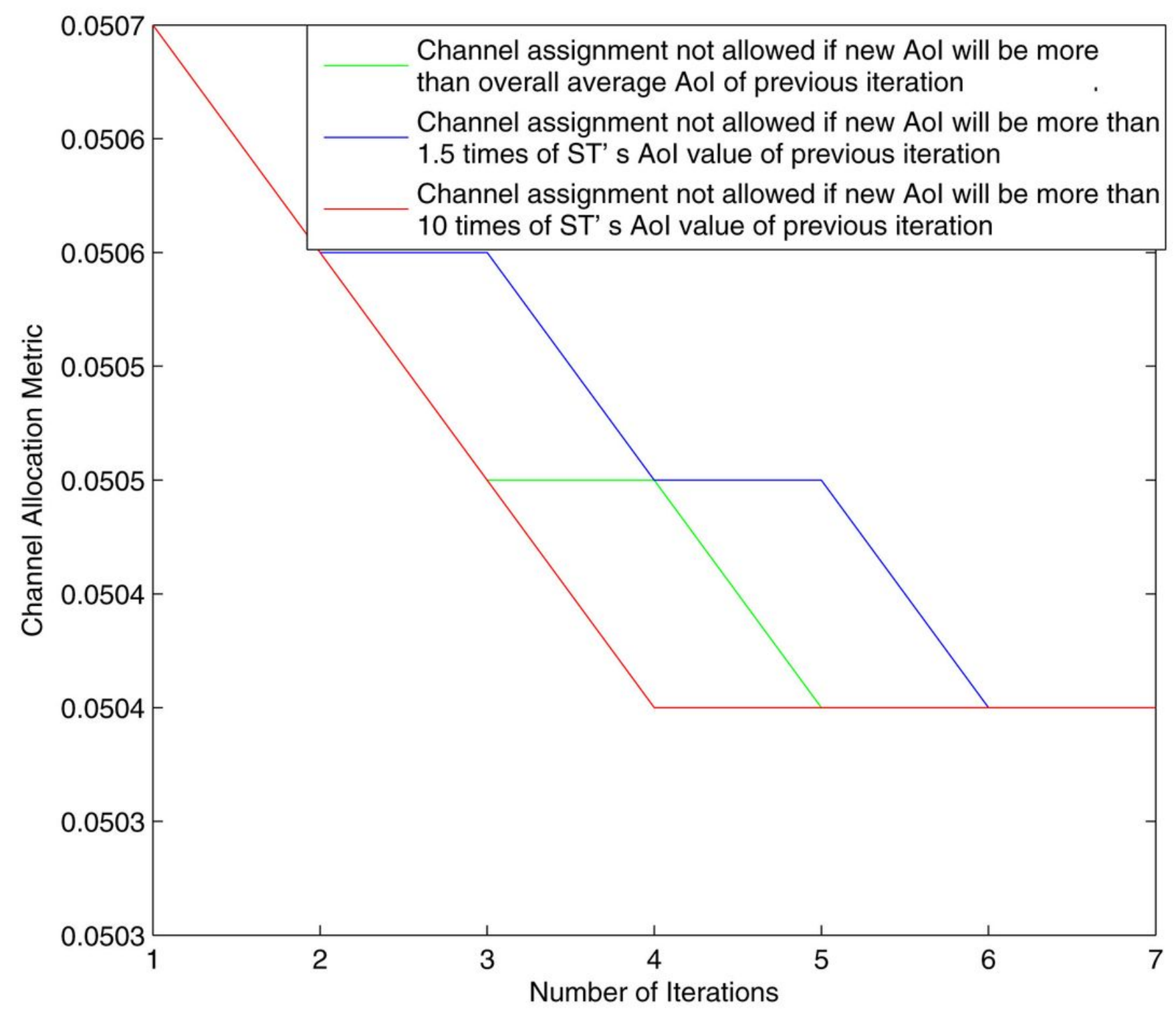

Figure 12

Channel Distribution Performance within increasing time slots.

\section{Supplementary Files}

This is a list of supplementary files associated with this preprint. Click to download.

- Biography.docx 